\title{
Les modèles d'ajustement des courbes de lactation
}

\author{
Sylvie MASSElin, D. SAUVANT, P. CHAPOUTOT, D. MILAN (*) \\ INRA, Département des Sciences animales et Station de Nutrition et Alimentation \\ I.N.A. Paris-Grignon, 16, rue Claude-Bernard, F 75231 Paris Cedex 05 \\ * INRA, Laboratoire de Génétique Cellulaire, Centre de Recherches de Toulouse \\ B.P. 27, F 31326 Castanet Tolosan Cedex
}

\begin{abstract}
Résumé
L'intérêt que présente la courbe de lactation des femelles de ruminants se traduit par une multiplicité des modèles mathématiques proposés pour la décrire ou la prévoir.

Les modèles déterministes, linéaires ou non, représentent la grande majorité des propositions publiées dans la bibliographie. Certains d'entre eux permettent d'obtenir de bons ajustements de tout ou partie de la courbe de lactation, ainsi que d'estimer certaines caractéristiques classiques de la courbe (date et niveau du maximum de production, persistance en phase décroissante, production totale). Cependant, l'interprétation des paramètres des modèles les plus complexes est difficile et, dans ces cas, il est impossible de les relier de façon simple à ces caractéristiques classiques. Parmi les modèles déterministes, la fonction Gamma, proposée par Wood (1967) a été la plus communément employéc dans les études portant sur la courbe de lactation. Le succès de ce modèle non linéaire s'explique, d'une part parce qu'il prend en compte la phase ascendante de la courbe, et d'autre part, parce que les paramètres sont faciles à estimer après linéarisation de réquation. Les modèles déterministes d'ajustement traduisent assez fidèlement la courbe, par conséquent, ils peuvent être d'un usage intéressant pour l'interprétation de résultats d'expérimentation. Mais ils ne sont pas aptes à rendre compte logiquement des variations à court terme du niveau de production autour de la tendance générale. Pour traduire ces variations, quelques auteurs ont cherché à construire des modèles d'ajustement de la courbe de lactation, dits stochastiques, qui s’appuient sur un modèle déterministe de description de l'allure globale de la courbe, et une fonction traduisant le caractère autocorrélatif de la production laitière. De tels modèles ouvrent des perspectives nouvelles. notamment pour l'analyse des résultats du contrôle laitier.

Enfin, il faut souligner que dans la majorité des cas, les propositions de modèles de description et/ou de prédiction de la production laitière sont empiriques et ne cherchent pas à rendre compte des phénomènes nutritionnels et physiologiques sous-jacents à la sécrétion lactée. Cette lacune pourraît être en partie comblée par le développement de recherches en matière de modélisation des systèmes biologiques, aujourd'hui facilitées par l'existence d'outils informatiques performants.
\end{abstract}

Mots clés : lactation, courbe, modèle, estimation, prédiction. 


\section{Introduction}

La courbe de lactation des femelles de ruminants, principalement dans les espèces bovine et caprine, a focalisé l'attention des chercheurs (généticiens, nutritionnistes, physiologistes, technologues,...), des agents de développement, des économistes et industriels de la filière lait. L'intérêt considérable porté par des spécialistes de disciplines différentes à la courbe de lactation, tant comme moyen de prévision que comme élément de diagnostic, s'explique, d'une part, par le rôle déterminant de la production laitière vis-à-vis du revenu de l'activité d'élevage correspondante et d'autre part, par les nombreux facteurs biologiques suceptibles d'en modifier le déroulement.

De nombreux travaux ont eu pour but d'ajuster mathématiquement les courbes de lactation. Aucune synthèse n'ayant été à notre connaissance publiée récemment sur cet aspect, une étude critique comparative des différents modèles publiés a été entreprise pour aider les différents utilisateurs dans leur choix et pour tenter d'orienter les recherches futures.

\section{Les caractéristiques de la courbe de lactation}

Au cours de la lactation et en l'absence de tout facteur limitant, la production de lait brute (PL (t)), suit, en fonction du temps $t$, une évolution généraie rappelée figure 1. Elle augmente durant les premières semaines suivant la mise-bas, atteint un maximum, puis décroît progressivement jusqu'au tarissement. La courbe de lactation peut être caractérisée par un certain nombre de paramètres :

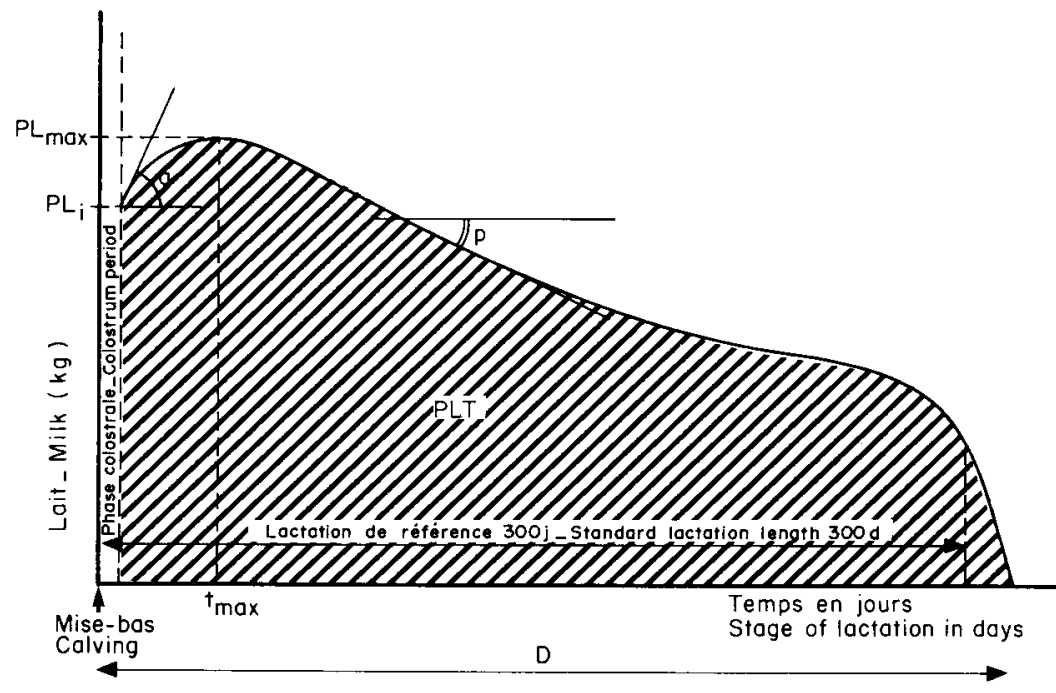

FIG. 1

Représentation schématique de la courbe de lactation de la vache laitière (DELAGE, LEROY \& POLY, 1953).

General shape of the dairy cow lactation curve. 
- la durée de la lactation (D) définie par l'intervalle mise-bas-tarissement ;

- la production totale (PLT) obtenue en cumulant les productions laitières quotidiennes correspond à l'intégrale de la courbe de lactation (surface hachurée sur la fig. 1) ;

- la production initiale $\left(\mathrm{PL}_{\mathrm{i}}\right)$ estimée par la moyenne des productions des $4^{\mathrm{e}}, 5^{\mathrm{e}}$ et $6^{e}$ jour de lactation (Decaen, Calomiti \& Poutous, 1970 ; Hoden, 1978) ;

- la production journalière maximum $\left(\mathrm{PL}_{\mathrm{max}}\right)$ et la date à laquelle on observe ce maximum $\left(\mathrm{t}_{\mathrm{mix}}\right)$. Lorsqu'on dispose d'un modèle mathématique d'ajustement de la courbe de lactation, les quantités $P_{L_{\max }}$ et $t_{\max }$ sont obtenues respectivement comme ordonnée et abscisse du point où la dérivée de la courbe (dPL (t)/dt) s'annule ;

- le rythme de croissance en phase ascendante : Delage, Leroy \& Poly (1953) proposent de le mesurer par la tangente de l'angle a (fig. 1), correspondant à la dérivée de la courbe de lactation (dPL (t)/dt). Actuellement, la phase croissante est souvent caractérisée par l'écart entre $\mathrm{PL}_{\max }$ et la production initiale $\left(\mathrm{PL}_{\mathrm{i}}\right.$ ) (Decaen, Calomiti \& Poutons, 1970 ; Hoden, 1978). La quantité $\mathrm{PL}_{\max }$ est obtenue soit comme valeur la plus élevée des moyennes mobiles des productions laitières de 3 jours consécutifs, soit comme valeur la plus élevée des moyennes hebdomadaires;

- la persistance de la production en phase décroissante : elle est le plus souvent identifiée à une mesure de la décroissance de la production sur un intervalle de temps. La dérivée de la courbe de lactation $(\mathrm{P}=\mathrm{dPL}(\mathrm{t}) / \mathrm{dt})$ représente une mesure absolue de cette décroissance P (Delage, Leroy \& Poly, 1953, fig. 1). Il est également possible de mesurer la persistance relative $p$ de la production et de l'exprimer, par exemple, en pourcentage :

$$
p=100 .(d P L(t) / d t) / P L(t)
$$
taire :

En pratique, la persistance est fréquemment définie par la relation complémen-

$$
\mathrm{p}=100 .(1-(\mathrm{dPL}(\mathrm{t}) / \mathrm{dt}) / \mathrm{PL}(\mathrm{t})) \text {. }
$$

Différents auteurs ont utilisé d'autres méthodes d'appréciation de la persistance de la lactation, par exemple, le rapport de productions journalières consécutives $\operatorname{PL}(t+1)$ / PL (t) (Gooch, 1935 ; Ponteconvo, 1940), ou le rapport de la production totale en 305 jours à la production maximale (MADSEN, 1975). Dans plusieurs travaux (HUTH, 1971 ; Milojic \& Simovic, 1971 ; Madsen, 1975 ; Schneeberger, 1981), le critère retenu pour mesurer la persistance est un rapport des productions laitières cumulées pendant 2 périodes de la lactation, tel que :

$$
\mathrm{P}(2 \div 1)=\operatorname{PLT}(\text { jours } 101 \text { à 200)/PLT (jours } 1 \text { à 100) }
$$

ou :

$$
\mathrm{P}(3 \div 1)=\text { PLT (jours } 201 \text { à 300)/PLT (jours } 1 \text { à 100) }
$$

ou :

$$
\text { P }(3 \div 2)=\text { PLT (jours } 201 \text { à 300)/PLT (jours } 101 \text { à 200); }
$$

- la forme de la courbe : cette caractéristique, bien que souvent évoquée, n’a à notre connaissance pas donné lieu à définition ou quantification. La multiplicité des types de formes apparaît clairement lorsqu'un ensemble de courbes de lactation individuelles est traité par une méthode telle que l'analyse en composantes principales (Sauvant \& Fehr, 1976). Dans ce cas, la première composante principale exprime à travers un «facteur de taille » les différences individuelles de production totale (fig. 2 et 3). Par contre, les autres composantes discriminent, à production totale comparable, différents types de formes de courbes de lactation; ainsi la seconde composante 


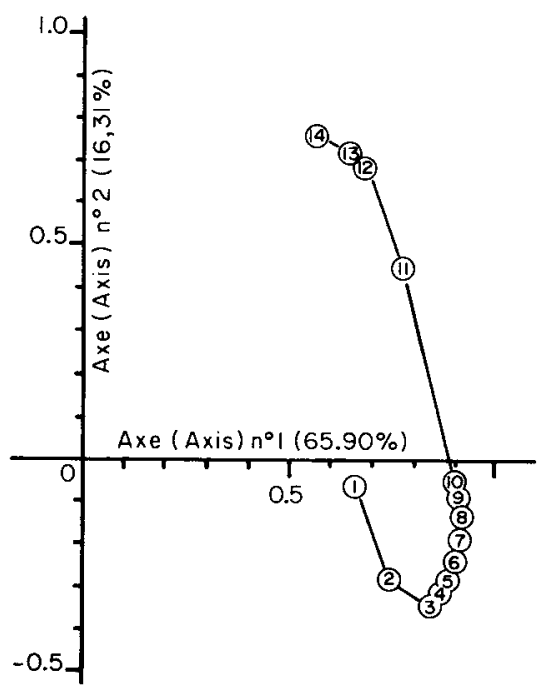

FIG. 2

Analyse en composantes principales de 60 courbes de lactation de chèvres : corrélations des productions moyennes par quinzaine (nombres entourés d'un cercle) avec les composantes 1 et 2.

Principal component analysis of 60 goat lactation curves : correlation between fortnight mean productions and the 2 first principal components (the circled numbers are the fortnight ones).

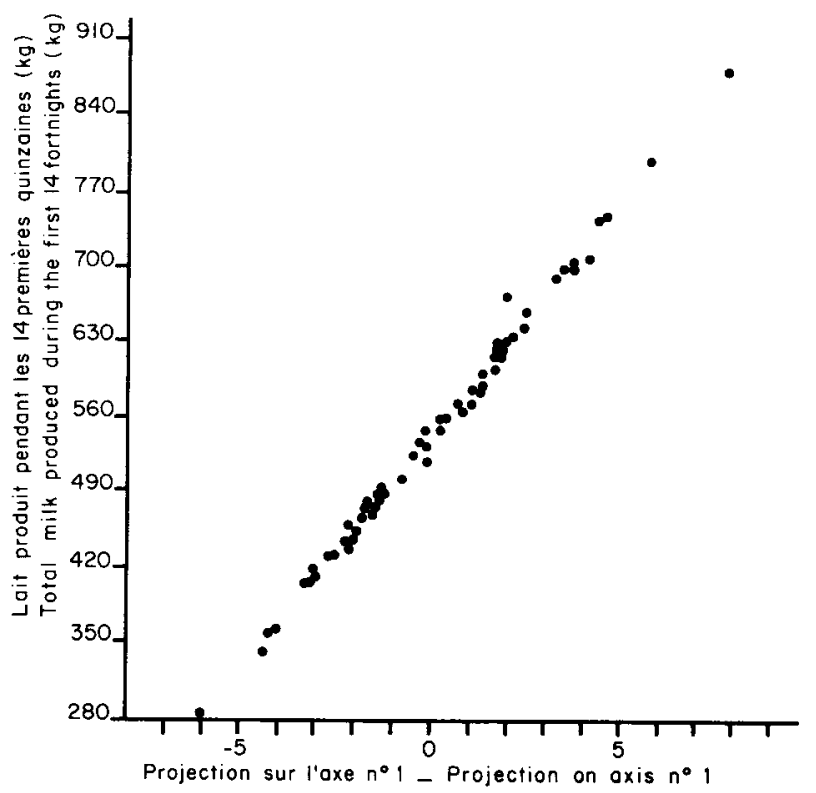

FIG. 3

Relation entre le niveau de production laitière totale des chèvres et leur abscisse sur la première composante principale de la figure 2.

Relationship between total milk yield of goats and their ordonate on the first principal component of figure 2. 
représentée par l'axe $\mathrm{n}^{\circ} 2$ de la figure 2 discrimine les animaux en fonction de la croissance entre la $1^{\text {re }}$ et la $3^{\mathrm{c}}$ quinzaine de production et en fonction de la persistance de la phase décroissante, en particulier entre la $10^{\mathrm{c}}$ et la $12^{\mathrm{e}}$ quinzaine de production (données non publiées). Les formes ainsi mises en évidence par les composantes d'ordre supérieur à 1 correspondent en fait à différentes combinaisons des valeurs de la croissance et de la persistance de la production de lait.

Dans la suite de cette étude, les paramètres des différents modèles proposés pour décrire la courbe de lactation seront, dans la mesure du possible, interprétés en fonction de ces 6 caractéristiques de la courbe de lactation. Le tableau 1 en résume les aspects essentiels.

\section{Les modèles d'ajustement linéaires}

La production laitière est considérée comme une combinaison linéaire du temps écoulé depuis la mise-bas, ou/et d'une fonction simple de celui-ci (puissance, logarithme...). L'estimation des paramètres pour l'ajustement du modèle à une courbe observée est alors immédiate par la méthode des moindres carrés. Plusieurs types de modèles linéaires ont été proposés.

\section{A. Modèles linéaires du premier degré}

Dans une étude portant sur la mesure de la persistance des courbes de lactation de vaches laitières, GAINES (1927b) propose l'équation :

$$
\text { PL }(t)=a-b . t
$$

comme modèle décrivant la décroissance de la production laitière brute durant la lactation. Dans cette équation, PL $(\mathrm{t})$ représente la production laitière, et $\mathrm{t}$ le temps écoulé depuis la mise-bas. Le paramètre a est un estimateur de la production initiale, $\mathrm{PL}_{\mathrm{i}}$, et $\mathrm{b}$, une mesure de la persistance absolue $\mathrm{P}$, supposée être constante au cours de la lactation (tabl. 1).

D'autres auteurs, tels que Fischer (1958) ont repris ce modèle, surtout afin de quantifier la persistance de la lactation. Delage, Leroy \& Poly (1953) l'ont testé sur 1250 lactations de vaches Frisonnes Pie Noire, dont ils ont retenu les productions entre le $60^{\mathrm{e}}$ et le $250^{\mathrm{c}}$ jour post-partum, pour le comparer à 3 autres modèles : régression hyperbolique, régression parabolique et régression exponentielle (voir plus loin).

OstergaARd (1979) a également utilisé ce modèle pour comparer les effets de 8 stratégies d'apport d'aliments concentrés sur les performances des vaches laitières. Le critère pris en compte dans cette étude est la persistance absolue $P$ de la lactation au cours des 36 premières semaines suivant la mise-bas, c'est-à-dire la valeur du coefficient b du modèle (1) pour chaque lot d'animaux.

Panicke \& Bode (1973) ont cité en introduction de leur article le modèle linéaire utilisé par Kwizda, Hopler \& Teufelhart (1957) pour décrire 769 courbes de production laitière de vaches :

$$
\text { PL }(t)=10^{-5} . \text { PLT. }(588,2-1,612 . t)
$$

avec PL $(\mathrm{t})$ : production journalière $(\mathrm{kg})$

$\mathrm{t}$ : stade de lactation en jours $(30<\mathrm{t}<365)$

PLT : niveau de production totale $(\mathrm{kg})$. 


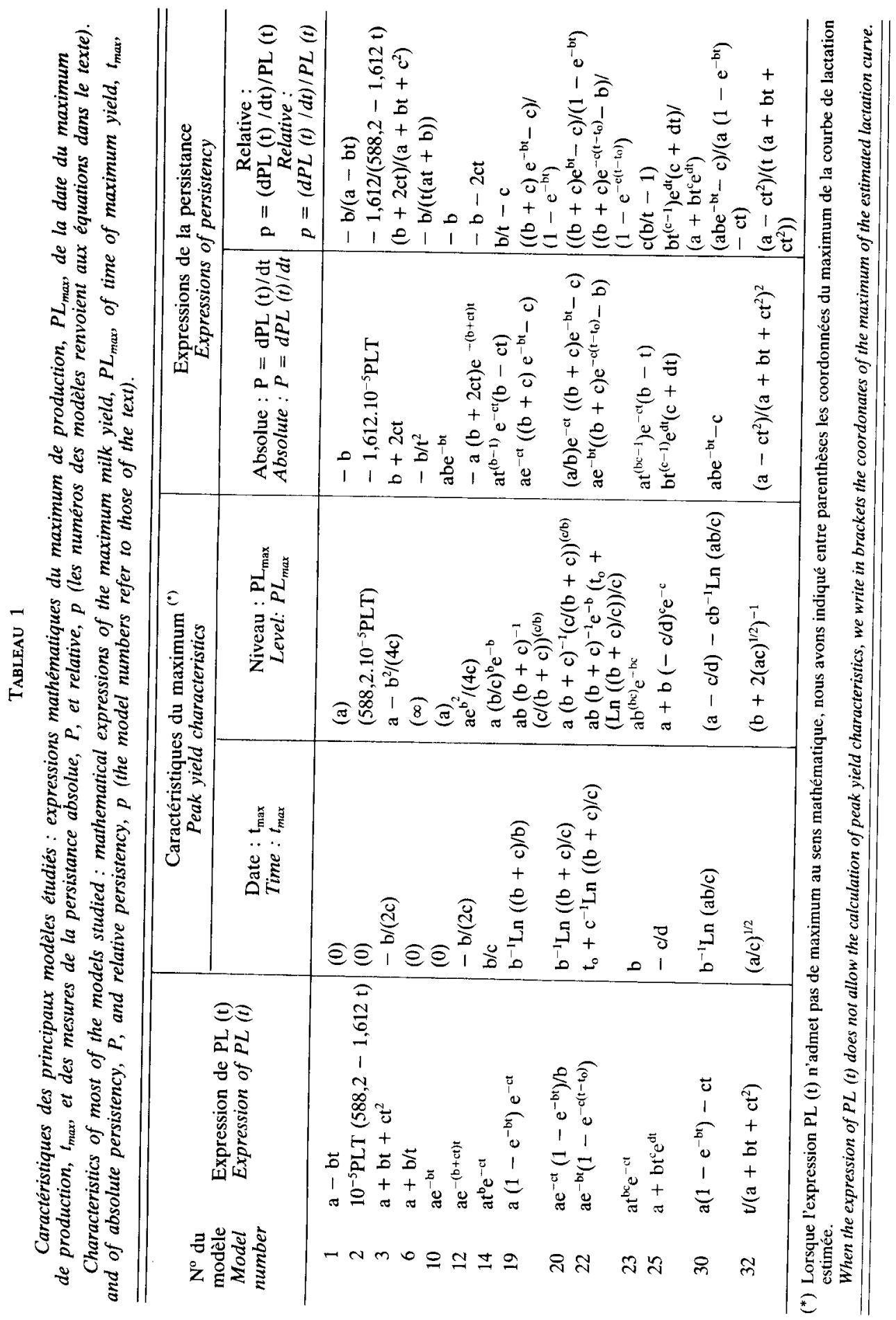


Dans ce modèle apparaissent les 2 paramètres dont Delage, Leroy \& Poly (1953) avaient fourni une interprétation : la production initiale théorique, $P_{L_{i}}$, estimée par $588,2.10^{-5}$.PLT, homogène au paramètre a de l'équation (1), et la persistance absolue $\mathbf{P}$ de la production laitière, estimée par la quantité $1,612 \cdot 10^{-5}$.PLT, exprimée en $\mathrm{kg} / \mathrm{j}$ et homogène au paramètre $b$ de l'équation (1).

Dans ce modèle, le niveau de production totale est considéré comme un facteur de variation de la forme de la courbe de lactation : plus le niveau de production totale est élevé, moins la persistance absolue $\mathrm{P}$ est bonne (tabl. 1) ; par contre, la décroissance relative $p$ de la production, qui est une fonction croissante du temps, comme dans le cas de l'équation (1), est indépendante du niveau de production totale PLT (tabl. 1). Le niveau de production totale s'avère avoir un effet significatif sur la forme de la courbe de lactation dans nombre de travaux (KwIzdA, Hopler \& TeufelharT, 1957 ; Huth, 1971 ; Zimmermann \& Sommer, 1973 ; Auran \& Mocquot, 1974 ; Congleton \& Everett, 1980b). Mais alors que Zimmermann \& Sommer (1973), comme Kwizda, Hopler \& Teufelhart (1957), constatent, lorsque la production totale s'accroît, une augmentation de la production initiale et une diminution de la persistance absolue $P$, estimées par leurs modèles linéaires du premier degré, Huth (1971) indique quant à lui une amélioration de la persistance, et une élévation du niveau de production initiale. Cependant, il convient de considérer avec prudence ces observations puisque HuTH (1971) estime la persistance de la production de lait par le rapport $\mathrm{P}(2 \div 1)$ défini plus haut.

Le modèle d'ajustement linéaire de la courbe de lactation a souvent été employé par les généticiens. Ainsi BonNier (1935) a caractérisé la décroissance de la production au cours des 13 premières semaines après la mise-bas par le coefficient de régression linéaire correspondant, ceci afin de comparer les lactations partielles des mères et des filles. Plus récemment, Gravert \& BaPTIST $(1973,1976)$ ont utilisé un modèle comparable à celui de l'équation (1) pour calculer l'héritabilité de la production initiale, $\mathrm{PL}_{\mathrm{i}}$, de la persistance absolue, $\mathrm{P}$, et de la production de lait totale en 305 jours, PLT, calculée par intégration.

\section{B. Polynômes de degré supérieur ou égal à 2}

\section{Modèle parabolique}

Dans ce cas l'équation d'ajustement est du type :

$$
\operatorname{PL}(t)=a+b . t+c . t^{2}
$$

avec PL $(\mathrm{t})$ : production laitière

$t$ : stade de lactation

$\mathrm{a}, \mathrm{b}, \mathrm{c}$ : paramètres du modèle.

Comme précédemment, le paramètre a peut être interprété comme un estimateur de la production initiale. Le paramètre $b$ traduit la croissance absolue initiale de la courbe, et le coefficient $c$, négatif pour que l'extremum soit un maximum, concourt à caractériser l'aplatissement de la courbe de lactation. La persistance absolue $\mathrm{P}$ décroît linéairement en fonction du temps (tabl. 1).

Delage, Leroy \& Poly (1953) ont obtenu des résultats d'ajustement satisfaisants avec ce modèle, mais ils ne l'ont appliqué qu'entre le $60^{\mathrm{e}}$ et le $250^{\mathrm{e}}$ jour de lactation (tabl. 2). Malhotra, Singh \& Singh (1980) comparent, à partir des lactations de 22 vaches Karan-Suisse, la qualité d'ajustement permise par 4 types de fonction (parabole, 
parabole plus un terme logarithmique, polynôme inverse, loi Gamma) et 3 rythmes de saisie des données (hebdomadaire, bimensuel, mensuel). Le modèle parabolique ne fournit pas le meilleur ajustement : le coefficient de corrélation multiple n'est supérieur à 0,8 que dans 5,10 et 16 cas sur 22 pour des données hebdomadaires, bimensuelles ou mensuelles respectivement, contre 5,17 et 20 cas sur 22 pour le modèle parabolique avec un terme logarithmique. Une des raisons du mauvais ajustement des données obtenu par la parabole est l'axe de symétrie passant par son sommet.

\section{Polynômes de degré 3}

Dans une étude portant sur les courbes de lactation de 298 chèvres laitières, SAUvant \& FeHR (1976) ont cherché à ajuster par un polynôme de degré 3 les productions des animaux :

$$
\text { PL (t) }=\mathrm{a}+\text { b.t }+ \text { c. } \mathrm{t}^{2}+\text { d. } \mathrm{t}^{3}
$$

avec PL $(\mathrm{t})$ : production de lait moyenne par quinzaine

$t$ : stade de lactation (numéro de quinzaine)

$\mathrm{a}, \mathrm{b}, \mathrm{c}, \mathrm{d}$ : paramètres du modèle.

Dans ce modèle, seul le paramètre a est facilement interprétable : il permet d'estimer la production initiale. Les 3 autres coefficients n'ont pas de signification biologique ou zootechnique. L'intérêt de la présence du terme de degré 3 par rapport au modèle parabolique est qu'il est susceptible d'introduire une dissymétrie dans la courbe.

Les résultats obtenus par Sauvant \& Fehr (1976) lors de l'ajustement des courbes, regroupées par numéro de lactation, montrent que les 4 paramètres, et donc le terme de degré 3 , sont dans tous les cas significatifs. Le coefficient de corrélation multiple varie de 0,595 pour les lactations de rang 3 à 0,639 pour les lactations de rang 1 (tabl. 2). Ces valeurs traduisent la médiocre qualité des ajustements, ce que confirme le coefficient de variation résiduelle, supérieur ou égal à $24 \%$ pour les 4 classes considérées de numéro de lactation.

\section{Polynômes de degré 5}

Pour prévoir les performances de vaches laitières, PANicke \& Bode (1973) ont étudié un modèle qui est du type polynôme de degré 5 par rapport au stade de lactation, et qui intègre les variations de production liées à l'âge de la vache à la misebas :

$$
\begin{aligned}
\text { PL }(t)= & a+\text { b.t }+ \text { c. } t^{2}+\text { d. } t^{3}+\text { e. } t^{4}+\text { f. } t^{5}+\text { g. } x^{3}+\text { h. } x^{2} \\
& + \text { i.x }+ \text { j.t.x }+ \text { k.t } t^{2} \cdot x+1 \cdot t^{3} \cdot x+\text { m.t. } x^{2}+\text { n.t. } .^{2} \cdot x^{2} \\
& + \text { p.t. } x^{3}
\end{aligned}
$$

avec PL ( $t)$ : production de lait, exprimée en proportion de la production moyenne d'une vache adulte entre le $15^{\mathrm{e}}$ et le $25^{\mathrm{e}}$ jour post-partum

$t$ : jour de lactation

$\mathrm{x}$ : âge au vêlage (mois)

$a, b, \ldots, p$ : paramètres du modèle.

La seule indication fournie par les autres quant à la qualité de l'ajustement est que l'erreur relative entre les productions observée et estimée, pour un âge au vêlage donné, n'excède pas $0,5 \%$. L'équation (5) semble donc permettre un ajustement de qualité satisfaisante de la courbe de lactation. 


\section{Autres modèles linéaires}

Delage, Leroy \& Poly (1953) ont employé un modèle d'ajustement hyperbolique de la forme :

$$
\text { PL }(t)=a+b \cdot t^{-1}
$$

avec PL $(\mathrm{t})$ : production laitière $(\mathrm{kg})$

$\mathrm{t}$ : stade de lactation (jours)

$\mathrm{a}, \mathrm{b}$ : paramètres du modèle.

pour ajuster les données de production laitière entre le $60^{\mathrm{e}}$ et le $250^{\mathrm{e}}$ jour de lactation. Cette équation leur a fourni l'ajustement le moins satisfaisant parmi les 4 modèles qu'ils ont étudiés (tabl. 2). En outre, une telle fonction ne peut s'appliquer à la totalité de la courbe de lactation puisqu'elle n'admet ni maximum, ni phase croissante.

Les auteurs ne proposent pas d'interprétation des 2 paramètres. Alors qu'il est difficile de donner une signification au paramètre $b$, le coefficient a fourni une estimation de la valeur limite de la production laitière en fin de lactation, sous l'hypothèse d'une durée de production infinie.

Decaen, Journet \& Poutous (1970), étudiant les 2 premiers mois de la lactation chez la vache laitière, ont essayé d'ajuster deux modèles aux données quotidiennes de production laitière et de taux butyreux, recueillies entre le $4^{\mathrm{c}}$ et le $63^{\mathrm{e}}$ jour de lactation :

$$
P L(t)=a+b . t^{2}+c . t^{1}+\text { d.t }+ \text { e. } t^{2}
$$

et :

$$
\operatorname{PL}(t)=a+b \cdot t^{-1}+c \cdot t^{-1} \cdot \operatorname{Ln} t
$$

avec PL (t) : production de lait ou taux butyreux

$\mathrm{t}$ : jour de lactation

Ln $\mathrm{t}$ : logarithme népérien de $\mathrm{t}$

$\mathrm{a}, \mathrm{b}, \mathrm{c}, \mathrm{d}, \mathrm{e}$ : paramètres des modèles.

La seconde équation a été proposée par Maymone \& Malossini (1959). Pour l'ajustement de la production de lait brute, Decaen, Journet \& Poutous (1970) concluent à la supériorité de l'équation (7) pour laquelle ils obtiennent un coefficient de corrélation multiple égal à 0,997 (tabl. 2), mais sans indiquer la valeur de ce coefficient pour l'équation (8).

Enfin Malhotra, Singh \& Singh (1980), en comparant 4 modèles de courbe de lactation, ont ajusté aux données de production laitière une expression du type :

$$
P L(t)=a+b . t+c . t^{2}+d \cdot \operatorname{Ln} t
$$

L'introduction du logarithme permet de rompre la symétrie du modèle parabolique et d'obtenir un ajustement d'une qualité excellente, le coefficient de corrélation multiple valant 0,991 pour des données hebdomadaires ou mensuelles, et 0,993 pour des données bimensuelles (tabl. 2). 


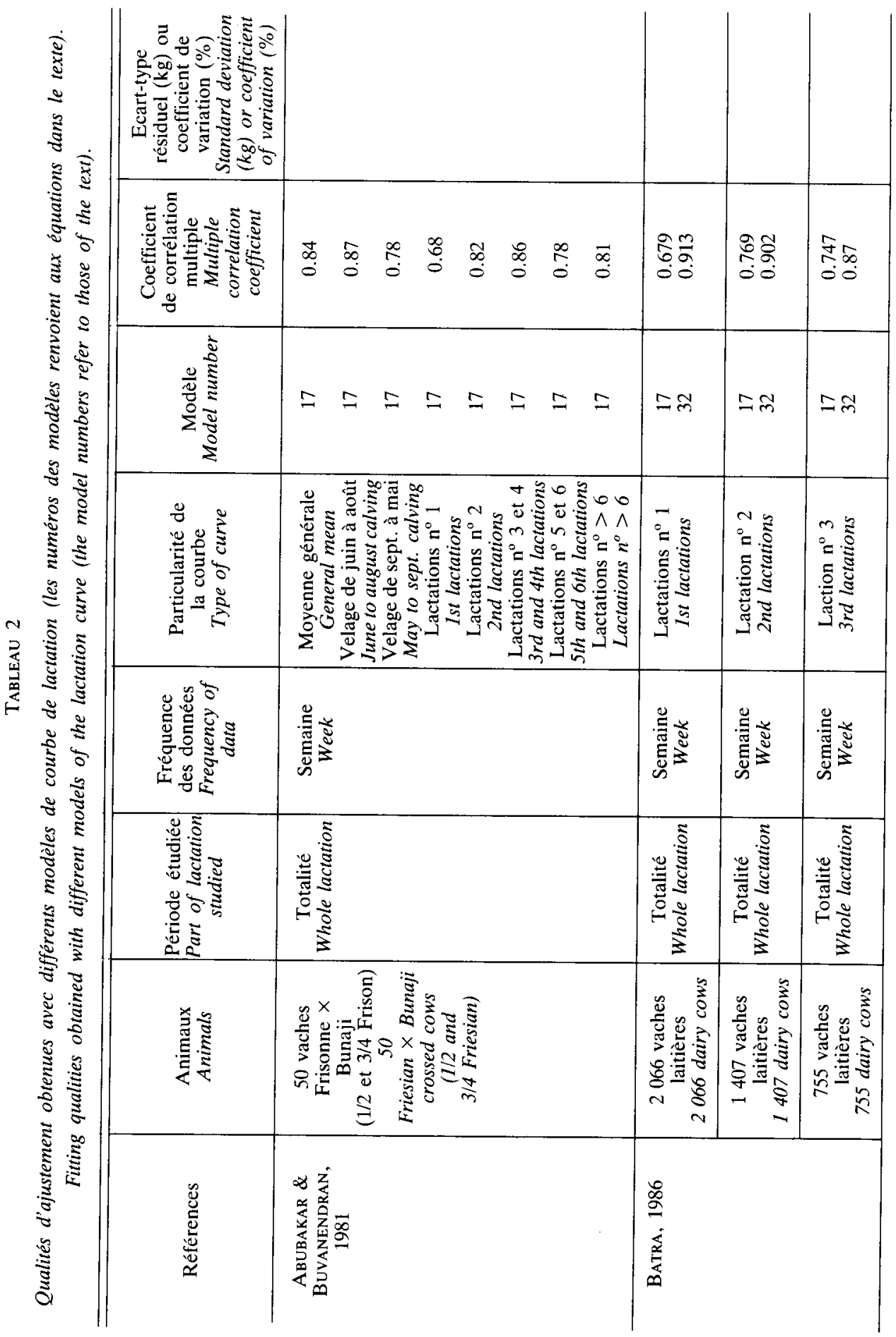




\begin{tabular}{|c|c|c|c|c|c|}
\hline 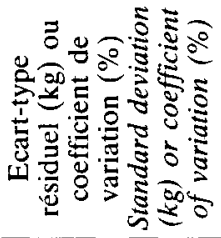 & 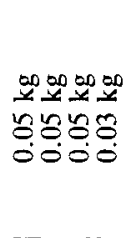 & 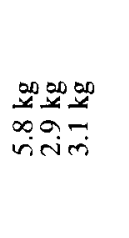 & & 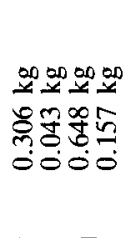 & \\
\hline 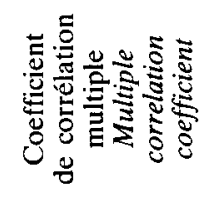 & 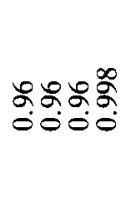 & 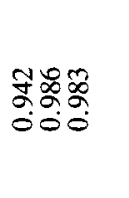 & 홍 & & 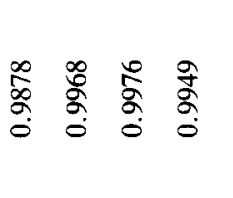 \\
\hline 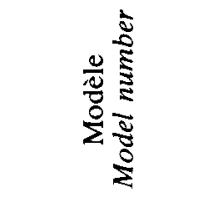 & N士ల్ల & 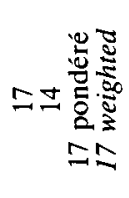 & $r$ & thoo & $\Xi \pm \Xi \Xi$ \\
\hline 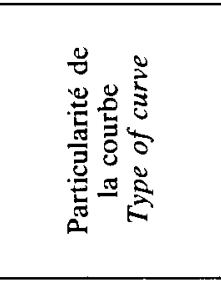 & 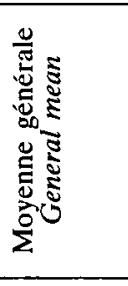 & & 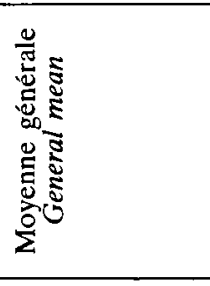 & 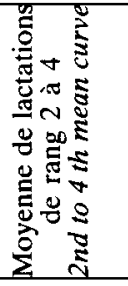 & 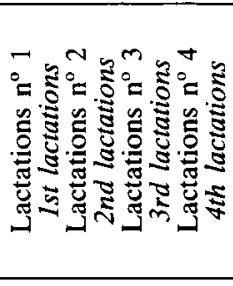 \\
\hline 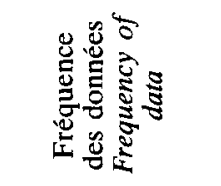 & 营 & 芯 & 亏亏 & 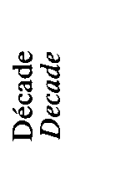 & \\
\hline 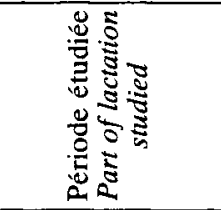 & 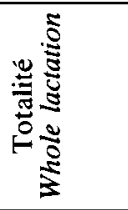 & 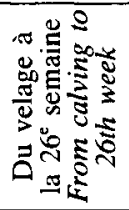 & 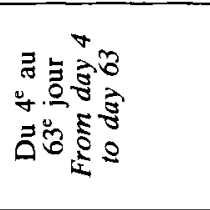 & 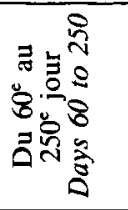 & 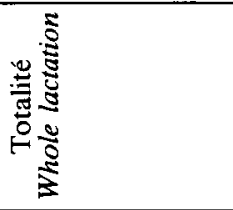 \\
\hline 兽昜 & 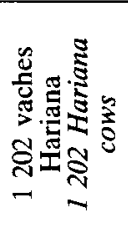 & 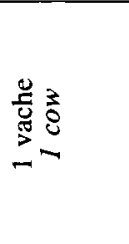 & 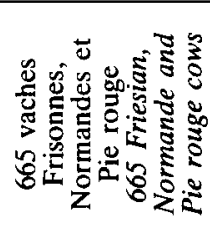 & 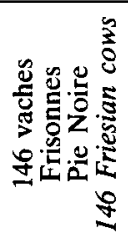 & 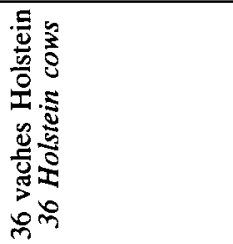 \\
\hline 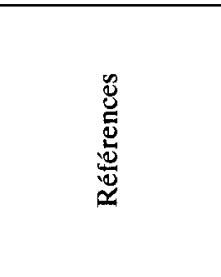 & 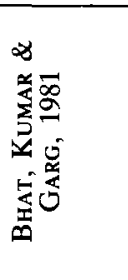 & 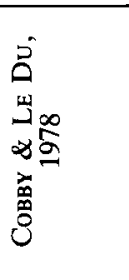 & 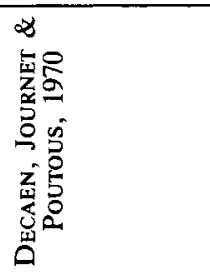 & 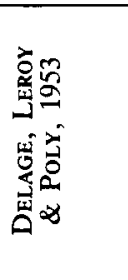 & 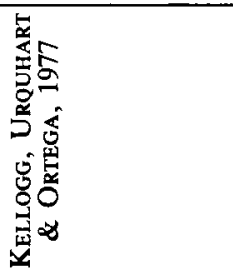 \\
\hline
\end{tabular}




\begin{tabular}{|c|c|c|c|c|c|c|}
\hline 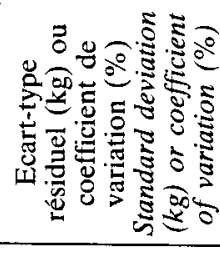 & & & 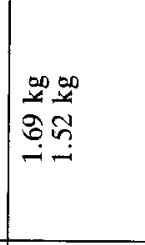 & & & \\
\hline 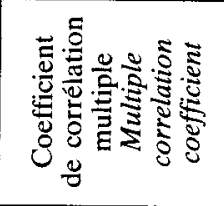 & 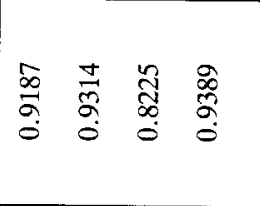 & 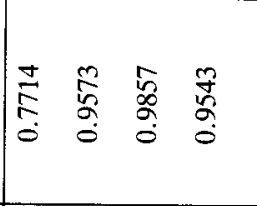 & 정 & $\vec{\sigma}$ & $\stackrel{\tilde{\sigma}}{\sigma}$ & 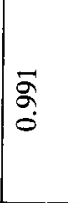 \\
\hline 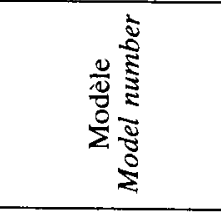 & $\stackrel{\Xi}{=} \pm$ & $\pm \quad \pm \quad \pm$ & $-\simeq$ & $a$ & $a$ & $a$ \\
\hline 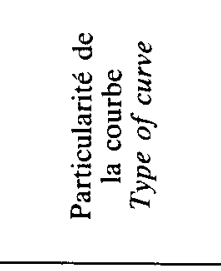 & 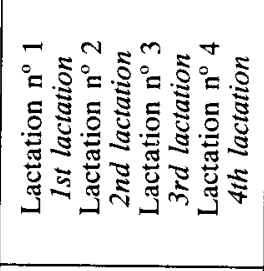 & 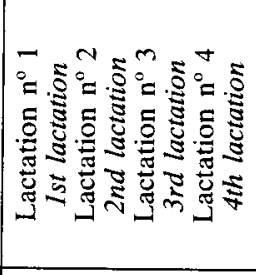 & 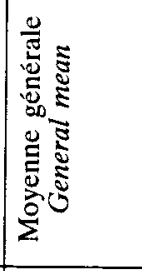 & 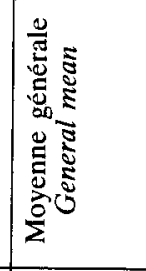 & 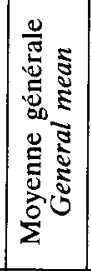 & 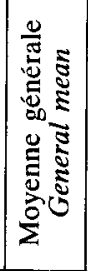 \\
\hline 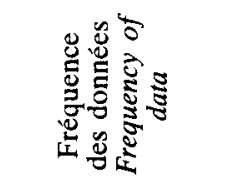 & & & 言 & 言 & 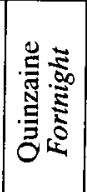 & $\frac{n}{2}$ \\
\hline 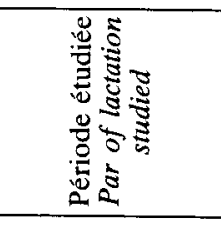 & 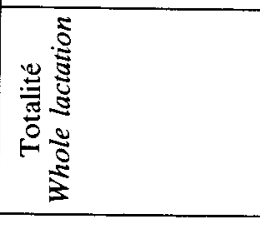 & 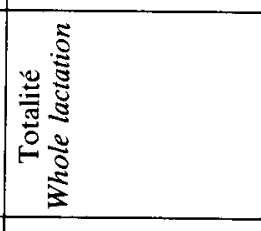 & 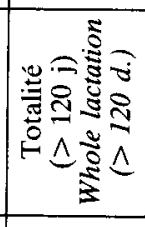 & 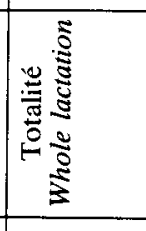 & & \\
\hline 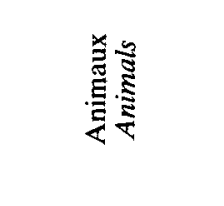 & 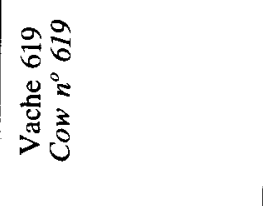 & 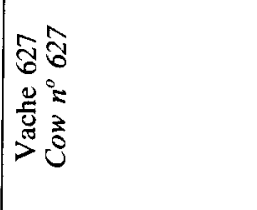 & 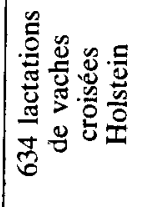 & 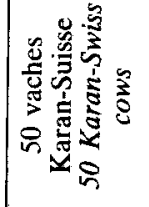 & & \\
\hline 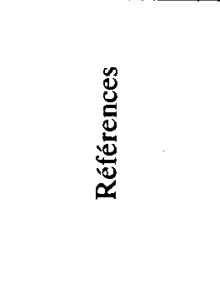 & 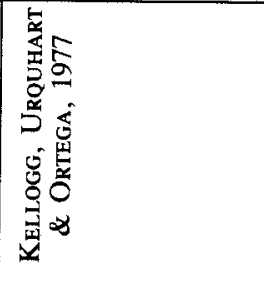 & & 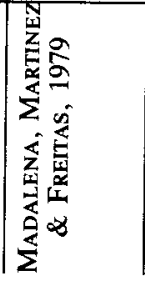 & 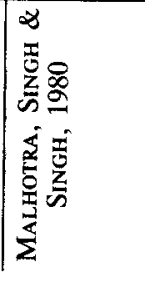 & & \\
\hline
\end{tabular}




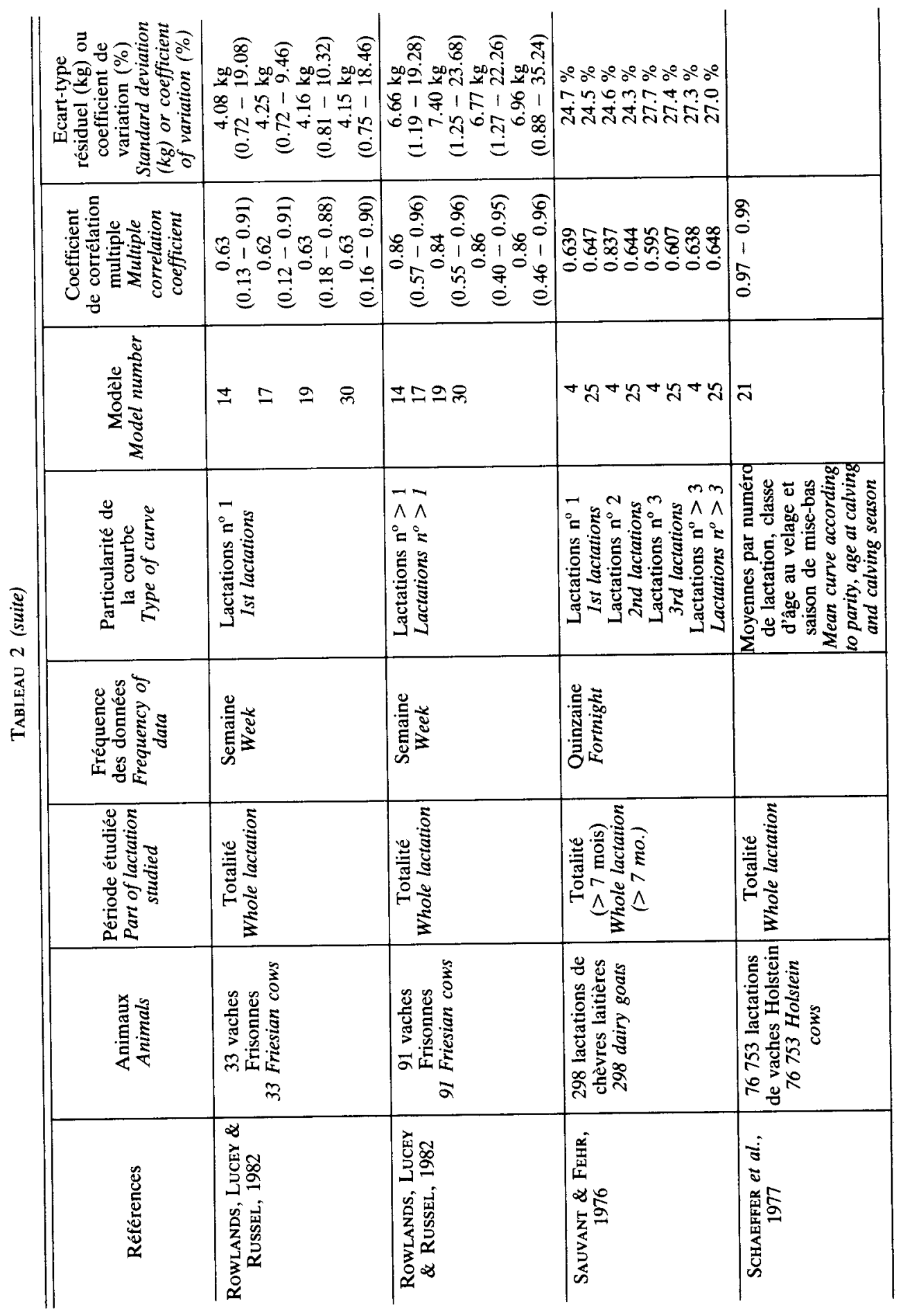




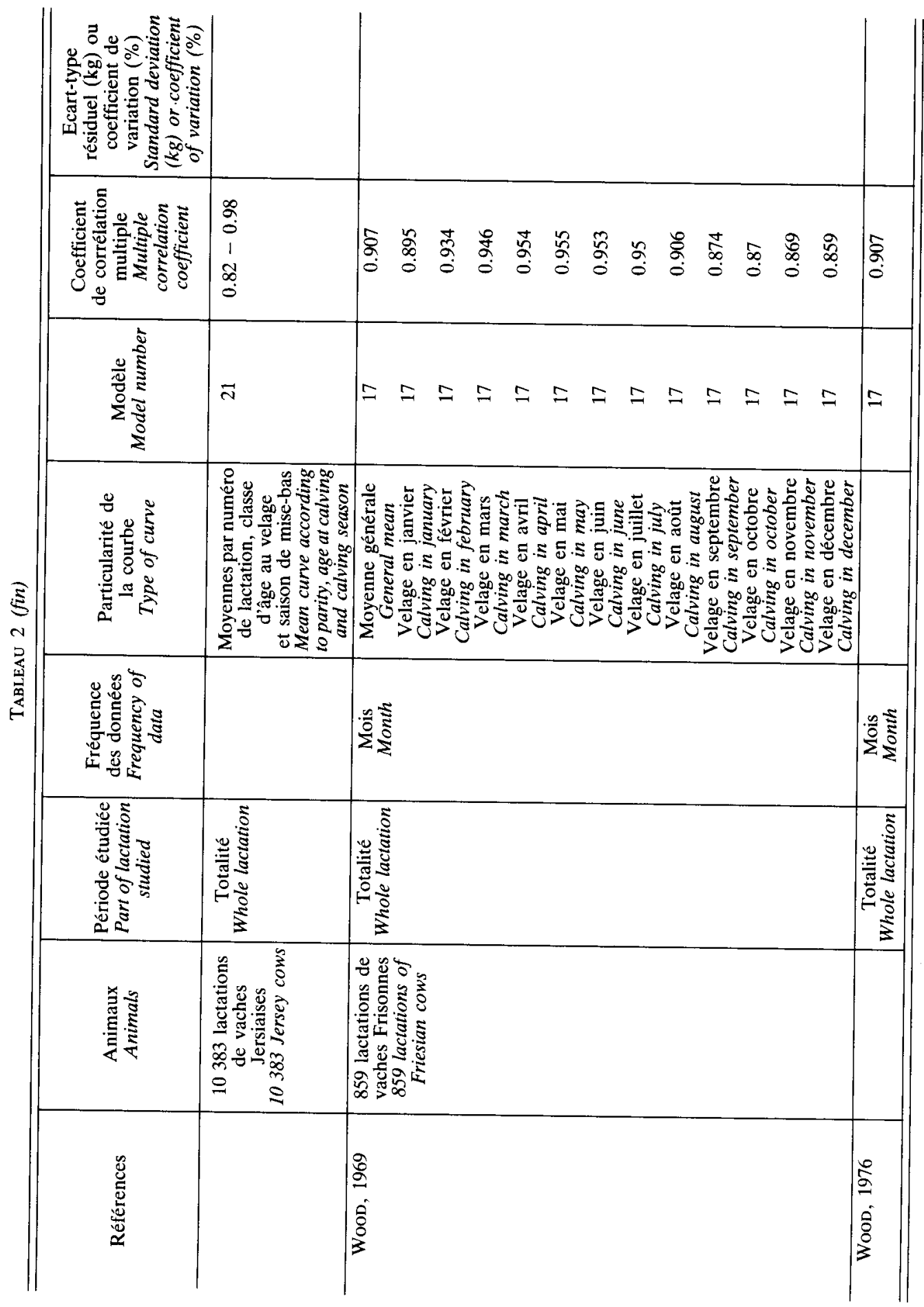




\section{Les modèles d'ajustement non linéaires}

Une partie des modèles rencontrés dans la bibliographie ne peuvent être considérés comme des fonctions linéaires des paramètres, ce qui nécessite l'emploi de méthodes d'estimation non-linéaires de ces derniers. De telles méthodes sont peu nombreuses et peuvent être classées en 3 catégories (Draper \& SMITH, 1981): des méthodes de linéarisation par développement de la fonction en série de Taylor, la méthode dite du " steepest descent ", l'algorithme de MARQuardT (1963), qui est un compromis entre les deux précédentes. Ce sont toutes des méthodes numériques itératives, coûteuses en temps de calcul.

Certains des modèles proposés peuvent cependant être linéarisés par une transformation mathématique simple de telle sorte que la méthode des moindres carrés linéaires peut encore être appliquée.

\section{A. Les modèles exponentiels}

\section{L'exponentielle simple}

Il s'agit, à notre connaissance, du plus ancien modèle proposé pour l'ajustement de la courbe de lactation (Brody, Ragsdale \& Turner, 1923 ; Turner, Ragsdale \& Brody, 1923 ; Brody, Turner \& Ragsdale, 1924 ; Gaines \& Davidson, 1926 ; GaInes, 1926, 1927a ; GoOCH, 1935 ; Pontecorvo, 1940) :

$$
\text { PL }(t)=a \cdot e^{-b . t}
$$

avec PL ( $t)$ : production laitière

$\mathrm{t}$ : stde de lactation

e : base des logarithmes népériens

$a, b$ : paramètres du modèle $(b>0)$.

Cette expression met en évidence la valeur b de la persistance relative (GaInes, 1927b). Actuellement, le contrôle laitier français signale à l'éleveur une chute de production de lait lorsque $b>0,1$ entre 2 contrôles successifs. Gooch (1935), utilisant une équation analogue évalue la persistance relative par la quantité $\mathrm{e}^{-b}$, et Ponteconvo (1940) propose comme coefficient de persistance de la lactation entre le $50^{\mathbf{e}}$ et le $240^{\mathrm{e}}$ jour post-partum une constante identique

$$
P=100 \cdot e^{-b}
$$

tirée de l'équation (10). L'intérêt pratique de ces 2 propositions d'expression de la persistance n'apparaît pas clairement ; il s'agit peut-être de l'attribution de la valeur 1 ou 100 au cas où la décroissance est nulle, et de valeurs comprises entre 1 et 0,9 ou $100 \%$ et $90 \%$ lorsque b est comrpis entre 0 et 0,1 .

Le modèle exponentiel simple comme le modèle linéaire simple, admet un maximum à l'instant initial. Par conséquent, il ne peut être employé que pour décrire la décroissance de la production laitière ou bien à la rigueur pour décrire la sécrétion des protéines ou des lipides qui est souvent maximale dès le démarrage de la lactation.

Les paramètres $\mathrm{a}$ et $\mathrm{b}$ sont faciles à estimer par la méthode des moindres carrés linéaires après une transformation logarithmique de l'expression (10):

$$
\operatorname{Ln} \operatorname{PL}(t)=\operatorname{Ln} a-b . t
$$


EDWARDS (1950) confirme l'intérêt de ce modèle en rapportant que la production laitière, exprimée en ordonnées semi-logarithmiques, diminue linéairement en fonction du temps durant la deuxième partie de la lactation. Le modèle exponentiel a également été employé par Singh \& Bhat (1978) et par Bhat, Kumar \& Garg (1981) pour ajuster des données de 44 semaines de lactation. La qualité des ajustements obtenue est intéressante avec, dans cette deuxième étude, un coefficient de corrélation multiple valant 0,96 et un écart-type résiduel de $0,05 \mathrm{~kg}$ (tabl. 2). On ne doit cependant pas s'en étonner car les vaches étudiées, de race Sahiwal, ont des niveaux de production laitière faibles, excédant rarement $10 \mathrm{~kg} / \mathrm{j}$, et leur courbe de lactation est décroissante dès la mise-bas.

\section{L'exponentielle parabolique}

SikKa (1950), remarquant que le taux de décroissance de la production laitière n'est pas constant, propose d'ajuster la phase décroissante de la courbe par un modèle de la forme :

$$
\operatorname{PL}(\mathrm{t})=\mathrm{a} \cdot \mathrm{e}^{-(\mathrm{b}+\mathrm{c} \cdot \mathrm{l}) \cdot \mathrm{t}}
$$

avec PL $(t)$ : production laitière

$t$ : stade de lactation

e : base des logarithmes népériens

$a, b, c$ : paramètres du modèle.

Le paramètre $b$ est la valeur de la décroissance relative $p$ au temps initial, et $c$, un indice de variation progressive du rythme de décroisssance par rapport à la valeur initiale b. Lorsque $c$ est positif, la décroissance relative va en s'accélérant alors que le phénomène inverse se produit lorsque $\mathrm{c}$ est négatif. La décroissance relative $\mathrm{p}$ de la production varie linéairement avec le stade de lactation (tabl. 1).

Comme dans le cas de l'exponentielle simple, les paramètres a, b et c sont faciles à estimer après transformation logarithmique de l'équation (12) :

$$
\operatorname{Ln} P L(t)=\operatorname{Ln} a-b . t-c . t^{2}
$$

Le modèle exponentiè parabolique peut théoriquement présenter un maximum pour l'instant :

$$
\mathrm{t}_{\max }=-\mathrm{b} /(2 . \mathrm{c})
$$

Ce maximum est obtenu pour une valeur de $t$ positive si les paramètres $b$ et $c$ sont tous deux différents de 0 et de signes contraires. Lorsqu'on ajuste des données de production de vaches dont les courbes de lactation ne présentent pas de pic, les valeurs estimées de b et $\mathrm{c}$ sont de signes contraires, indiquant que la date du maximum théorique se situe avant le vêlage. Ainsi les résultats de Bhat, Kumar \& Garg (1981), bien que permettant d'obtenir de très bons ajustements, avec un coefficient de corrélation multiple égal à 0,96 et un écart-type résiduel égal à $0,05 \mathrm{~kg}$, comme dans le cas de l'exponentielle simple (tabl. 2), estiment la date du maximum de production à environ 170 semaines avant le vêlage. 


\section{B. La fonction Gamma incomplète}

\section{Le modèle proposé par WooD}

Etant donné que les modèles exponentiels précités ne permettent pas de traduire correctement la phase de croissance de la lactation, Wood (1967) propose d'ajuster la totalité de la courbe par une fonction de type Gamma incomplète :

$$
\mathrm{PL}(\mathrm{t})=\mathrm{a} \cdot \mathrm{t}^{\mathrm{b}} \mathrm{e}^{-\mathrm{c} . \mathrm{t}}
$$

avec PL ( $\mathrm{t})$ : production laitière $(\mathrm{kg})$

$t$ : stade de lactation (semaines)

e : base des logarithmes népériens

$a, b, c$ : paramètres du modèle.

Dans cette expression, la fonction puissance $t^{\mathrm{h}}$ permet d'intégrer la phase ascendante de la lactation, alors que le terme exponentiel rend compte de la phase décroissante. Pour ces raisons, Wood (1967) interprète les paramètres b et c comme des indices d'intensité de croissance et de décroissance de la production respectivement, la fonction $t^{\mathrm{t}} . \mathrm{e}^{-\mathrm{c} . \mathrm{l}}$ apparaissant ainsi comme le «facteur forme » de la courbe. Le paramètre a est alors le facteur d'échelle du niveau de production, que WooD (1967) associe au niveau de production moyen du début de la lactation. Mais KREFT (1967) lui donne une signification légèrement différente, qui est d'estimer plutôt la quantité de lait totale produite pendant la lactation. Pour mettre en évidence cette interprétation, il écrit le modèle proposé par Wood sous la forme :

$$
\operatorname{PL}(t)=a \cdot P L T \cdot t^{b} \cdot e^{-c t}
$$

où le terme PLT représente la production de lait cumulée de la lactation.

A partir de l'ajustement individuel, limité à 20 semaines, de 54 courbes de lactation des vaches Françaises Frisonnes, nous avons confirmé que le coefficient a présente une corrélation plus forte avec le niveau de production des 5 premières semaines qu'avec celui des semaines suivantes. La même tendance apparaît pour les coefficients $b$ et $c$ bien que les corrélations entre les paramètres et les niveaux de production ne soient pas toutes significatives (fig. 4).

Wood $(1977,1979)$ a cherché à interpréter les paramètres a, b et c en fonction des flux d'énergie dans l'organisme. D'après cette approche, le paramètre a traduirait un potentiel de production qui est fonction de la capacité intrinsèque de sécrétion de la mamelle, du niveau des réserves mobilisables et des capacités d'ingestion et de diges-

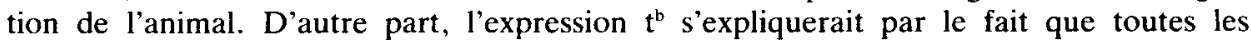
cellules sécrétrices ne seraient pas fonctionnelles aussitôt après la mise-bas. Ce dernier aspect devrait être, à notre sens, plutôt interprété comme la croissance de la capacité de l'organisme à assurer une néoglucogénèse permettant de synthétiser du lactose, qui est l'élément déterminant principal de la sortic de l'eau du lait. Enfin, le paramètre c intègrerait, d'une part, la baisse progressive de la contribution des réserves corporelles à la sécrétion lactée et, d'autre part, la décroissance exponentielle du nombre de cellules sécrétrices.

Partant de l'équation (14), Wood (1967) exprime la production laitière totale théorique de la lactation, PLT, par :

$$
\begin{aligned}
& \text { PLT }=a \cdot \int_{o}^{\infty} t^{b} \cdot e^{-c t} \cdot d t=a \cdot c^{-(b+1)} \cdot \Gamma(b+1) \\
& \text { avec } \Gamma(b+1)=\int_{0}^{\infty} t^{b} \cdot e^{-t} \cdot d t
\end{aligned}
$$




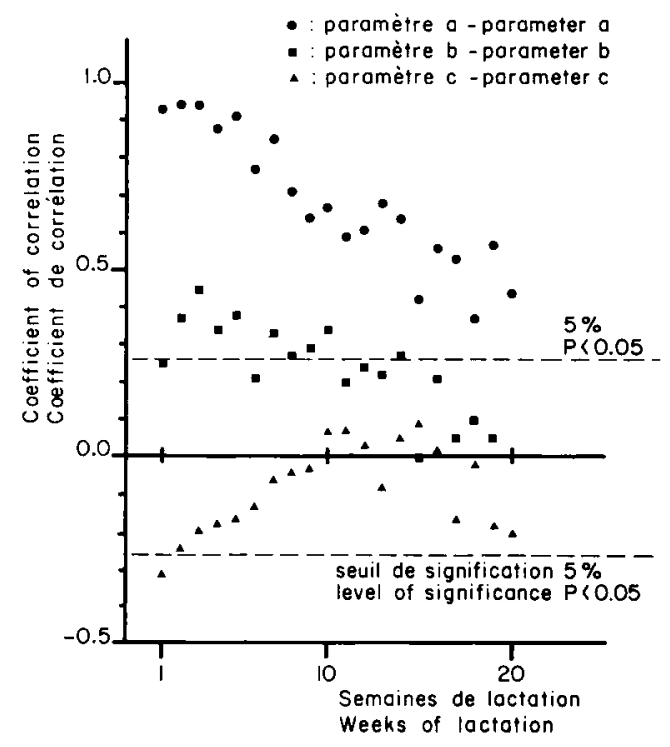

FIG. 4

Evolution des coefficients de corrélation entre les paramètres $a, b$ et $c$ du modèle de WOOD et les productions laitières au cours des 20 premières semaines de lactation (résultats de 54 courbes de lactation).

Evolution of correlation coefficients between WooD's model parameters and milk productions during the first 20 weeks of lactation (results of 54 lactation curves).

La courbe de lactation n'admettant pas de point d'inflexion au cours de sa phase ascendante, il est nécessaire que le paramètre b de l'équation (14) soit inférieur à 1 . Dans ces conditions, la quantité $\Gamma(b+1)$ est peu différente de 1 . Par conséquent, pour des courbes ayant un même niveau de production initiale, c'est-à-dire pour des valeurs comparables de a, c'est la quantité :

$$
S=c^{-(b+1)}
$$

qui conditionne l'estimation de la production laitière totale PLT. Effectuant une transformation logarithmique de cette expression, Wood (1967) propose de mesurer la persistance de la lactation par:

$$
\mathrm{s}=-(\mathrm{b}+1) \cdot \operatorname{Ln} \mathrm{c}
$$

Les variables $S$ et $s$, n'ayant pas d'unités, permettent seulement de comparer les niveaux de production totale, PLT, de plusieurs courbes de lactation, à condition qu'elles aient des niveaux de production initiale, $\mathrm{PL}_{\mathrm{i}}$, voisins. Ainsi, comme le soulignent Rao \& Sundaresan (1979), la persistance de la lactation serait d'autant plus forte que la valeur de $s$ est élevée. Cependant, il nous semble nécessaire d'employer ces estimateurs de la persistance avec prudence puisque leur calcul repose sur l'hypothèse d'une durée de lactation infinie, qui ne correspond à aucune situation réelle d'élevage. Par ailleurs, les expressions de $\mathrm{S}$ et de $\mathrm{s}$ sont issues de l'intégration de l'équation (14) et ne sont donc comparables ni à la décroissance absolue $P$, ni à la décroissance relative $\mathrm{p}$ de la courbe, toutes deux obtenues par le calcul de la dérivée de la courbe de lactation (tabl. 1). 
A partir de l'équation (14), Wood (1967) calcule également des estimations de la date du pic de production :

$$
\mathrm{t}_{\max }=\mathrm{b} / \mathrm{c}
$$

et du niveau de production maximale correspondant :

$$
\mathrm{PL}_{\max }=\mathrm{a} .(\mathrm{b} / \mathrm{c})^{\mathrm{b}} \cdot \mathrm{e}^{-\mathrm{b}}
$$

Le modèle (14) est souvent repris, sous l'appellation «modèle de WOOD », dans les travaux concernant la courbe de lactation (MADSEN, 1975; ShImIZU \& UMrod, 1976, 1977 ; Kellogg, Urquhart \& Ortega, 1977 ; Killen \& Keane, 1978 ; Madalena, Martinez \& Freitas, 1979 ; Rao \& Sundaresan, 1979 ; Congleton \& Everett, 1980a, b; Abubakar \& Buvanendran, 1981 ; Schneeberger, 1981; Rowlands, Lucey \& Russel, 1982). Il a été également utilisé comme équation de prédiction de la production de lait dans des travaux de simulation de systèmes d'alimentation des vaches laitières (Forbes, 1977 ; Wood, 1979 ; De Assis \& France, 1983 ; Bruce, Broadbent \& TopPs, 1984 ; Congleton, 1984) et dans des études sur l'ajustement des courbes de production d'œufs (GAvora et al., 1982).

Malgré les valeurs parfois assez élevées des coefficients de corrélation multiple obtenus avec le modèle de Wood, celui-ci présente un biais statistique important. En effet, bien que ce modèle s'écrive avec 3 paramètres, il semble que dans de nombreux cas, 2 pourraient peut-être suffire à déterminer la forme de la courbe en raison des corrélations qui existent entre les paramètres, b et $\mathrm{c}$ en particulier. Ainsi DhanoA (1981), ajustant 11 courbes de lactation de vaches Frisonnes a obtenu dans tous les cas un coefficient de corrélation supérieur à 0,9 entre ces 2 paramètres. De même, nous avons obtenu une valeur de corrélation de $+0,49(\mathrm{P}<0,01)$ entre $\mathrm{b}$ et $\mathrm{c}$, de $-0,32$ $(\mathrm{P}<0,05)$ entre a et $\mathrm{b}$, et de $+0,32(\mathrm{P}<0,05)$ entre a et $\mathrm{c}$, à partir des résultats d'ajustement de 54 courbes de lactation de vaches Frisonnes (tabl. 3).

\section{TABLEAU 3}

Matrice des corrélations intra-modèle et inter-modèle entre les paramètres du modèle de WooD et ceux du modèle de COBвY \& LE DU (données non publiées).

Within model and between model correlation coefficients between the parameters

\begin{tabular}{|c|c|c|c|c|c|c|c|}
\hline & & \multicolumn{3}{|c|}{$\begin{array}{l}\text { Modèle de Woon } \\
\text { Woon's model }\end{array}$} & \multicolumn{3}{|c|}{$\begin{array}{c}\text { Modèle de CoBry \& Le Du } \\
\text { CoBbY \& LE Du's model }\end{array}$} \\
\hline & & a & b & $\mathrm{c}$ & a & b & c \\
\hline $\begin{array}{l}\text { Modèle de Wood } \\
\text { WooD's model }\end{array}$ & $\begin{array}{l}\mathrm{a} \\
\mathrm{b} \\
\mathrm{c}\end{array}$ & $\begin{array}{c}1 \\
-0,32^{* *} \\
0,32 * *\end{array}$ & $\begin{array}{l}1 \\
0,49^{* *}\end{array}$ & & & & \\
\hline $\begin{array}{l}\text { Modèle de CoBBY \& LE Du } \\
\text { COBBY \& LE DU's model }\end{array}$ & $\begin{array}{l}\mathrm{a} \\
\mathrm{b} \\
\mathrm{c}\end{array}$ & $\begin{array}{l}0,85^{* *} \\
0,37^{* *} \\
0,12^{\text {ns }}\end{array}$ & $\begin{array}{r}0,03 \mathrm{~ns} \\
-0,57^{* *} \\
-0,07 \mathrm{~ns}\end{array}$ & $\begin{array}{c}0,51^{* *} \\
-0,28 * \\
0,02 \mathrm{~ns}\end{array}$ & $\begin{array}{l}1 \\
0,00 \mathrm{~ns} \\
0,04 \mathrm{~ns}\end{array}$ & $\begin{array}{c}1 \\
-0,05 \mathrm{~ns}\end{array}$ & 1 \\
\hline
\end{tabular}
of WOOD's model and COBBY \& LE Du's one (unpublished data).

Niveaux de signification/Levels of significance

** : Significatif au seuil de $1 \% /$ Significant at $P<0.01$

* : Significatif au seuil de $5 \% /$ Significant at $P<0.05$

ns : Non significatif/Not significant. 


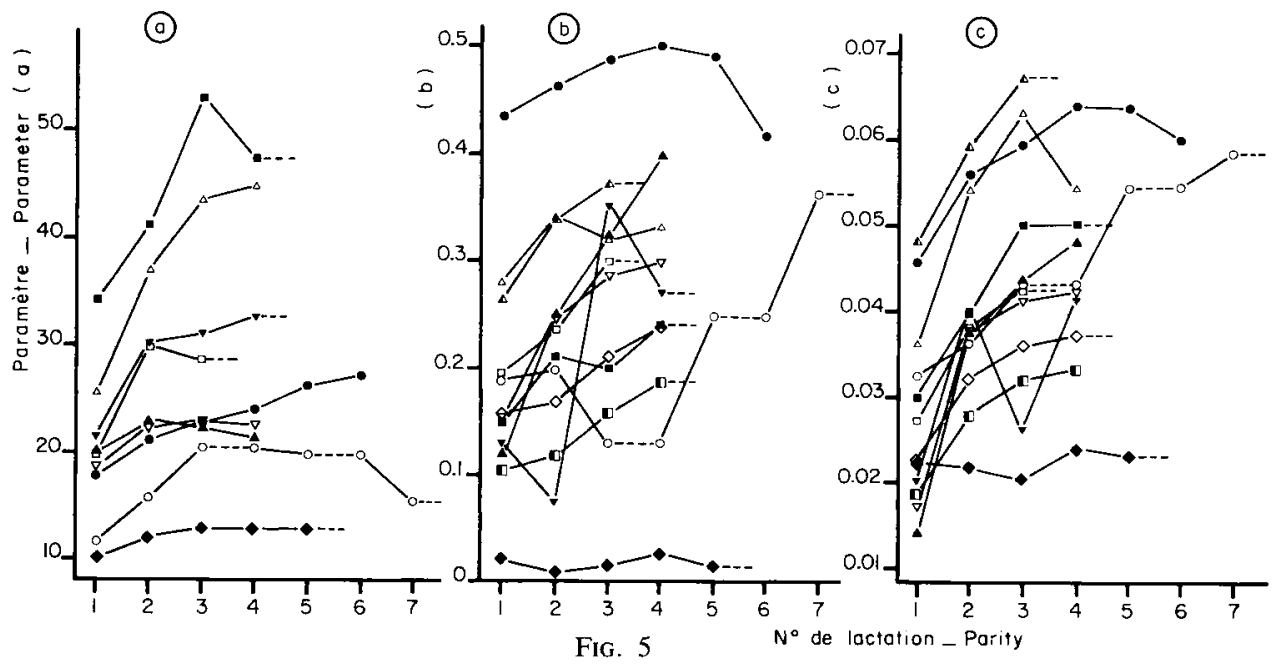

Variations des paramètres du modèle de Woos en fonction du numéro de lactation.

Variations in parameters of Woon's model according to parity.

Moyenne par numéro de lactation des valeurs des 3 paramètres obtenus par l'ajustement de 54 courbes individuelles de vaches Frisonnes (Sauvant et al., non publié).

Mean values according to parity, of the 3 parameters of 54 individual curves of Holstein cows.

Estimation par analyse de variance sur les 3 paramètres des courbes individuelles de 50 vaches croisées Frisonnes (Abubakar \& Buvanendran, 1981).

Estimation by analysis of variance of the 3 parameters of individual curves of 50 Friesian crossbred cows.

Estimation par analyse de variance sur les 3 paramètres de 169312 courbes individuelles de vaches Holstein (Congleton \& EveretT, 1980b).

Estimation by analysis of variance of the 3 parameters of 169312 individual curves of Holstein cows.

$\Delta$ Moyennes par numéro de lactation des valeurs des 3 paramètres obtenus par l'ajustement de 36 courbes individuelles de vaches Holstein (Kellogg, Urquhart \& OrTega, 1977).

Mean values according to parity, of the 3 parameters of 36 individual curves of Holstein cows.

$\nabla$ Valeurs obtenues par l'ajustement de la courbe moyenne correspondant à chaque numéro de lactation, obtenue à partir des courbes individuelles de 36 vaches Holstein (KellogG, UrQuHarT \& ORTEGA, 1977).

Values of the 3 parameters for mean curves according to parity, obtained from 36 individual curves of Holstein cows.

$\triangle$ Estimation par analyse de variance sur les paramètres b et c (KILlen \& Keane, 1978).

Estimation of parameters $b$ and $c$ by analysis of variance.

- Estimation par analyse de variance sur les 3 paramètres de 634 courbes individuelles de vaches croisées Frisonnes (Madalena, Martinez \& Freitas, 1979).

Estimation by analysis of variance, of the 3 parameters of 634 individual curves of Friesian crossbred cows.

- Estimation par analyse de variance sur les 3 paramètres de 1202 courbes individuelles de vaches Hariana (Singh, Bhat \& Kumar, 1979).

Estimation by analysis of variance of the 3 parameters of 1202 individual curves of Hariana cows. 
- Estimation par analyse de variance sur les 3 paramètres de 859 courbes individuelles de vaches Frisonnes (Wood, 1969).

Estimation by analysis of variance of the 3 parameters of 859 individual curves of Friesian cows.

$\triangle$ Estimation par analyse de variance sur les 3 paramètres de 1567 courbes individuelles de vaches laitières (WOOD, 1970).

Estimation by analysis of variance of the 3 parameters of 1567 individual curves of dairy cows.

$\diamond$ Estimation par analyse de variance sur les paramètres $\mathrm{b}$ et $\mathrm{c}$ obtenus par l'ajustement des courbes individuelles de vaches Holstein (WoOD, 1980).

Estimation by analysis of variance of parameters $b$ and $c$ of individual curves of Holstein cows.

D Estimation par analyse de variance sur les paramètres b et $c$ obtenus par l'ajustement des courbes individuelles de vaches Jersiaises (Woon, 1980).

Estimation by analysis of variance of parameters $b$ and $c$ of individual curves of Jersey cows.

Les points reliés par des pointillés indiquent que les numéros de lactation correspondant ont été regroupés dans l'étude.

The doted lines associate parities which have been studied together.

\section{Choix d'une méthode d'estimation des paramètres}

Un avantage essentiel du modèle de Wood est que l'équation (14) peut être linéarisée par transformation logarithmique :

$$
\operatorname{Ln} \text { PL }(t)=\operatorname{Ln} a+b . \operatorname{Ln} t-c . t .
$$

et il devient ainsi possible de calculer des estimations des paramètres par la méthode des moindres carrés linéaires.

C'est sous cette forme «log-linéaire » que les paramètres ont été estimés dans de nombreux travaux (Wood, 1967, 1968, 1969, 1972 ; MAdSen, 1975 ; Shimizu \& UMrod, 1976, 1977 ; Kellogg, Urquhart \& Ortega, 1977 ; Killen \& Keane, 1978 ; Madalena, Martinez \& Freitas, 1979, Rao \& Sundaresan, 1979 ; Congleton \& Everett, 1980a, b ; Abubakar \& Buvanendran, 1981 ; SCHNEeberger, 1981 ; RowlandS, Lucey \& Russel, 1982). Cependant cette méthode introduit des biais dans l'estimation des paramètres. En premier lieu, les variables $(t)$ et $(\operatorname{Ln} t)$ ne sont pas indépendantes, ce qui peut expliquer en partie les fortes corrélations observées entre les paramètres $b$ et $c$ dans nos travaux (tabl. 3) et dans ceux de Dhanoa (1981). Par ailleurs, CobBy \& Le Du (1978) ont posé la question du biais lié à la transformation logarithmique. En effet, les écarts entre valeurs observées et valeurs estimées dans le cas de la transformation logarithmique de l'équation (14) ne sont pas distribués aléatoirement. Ainsi, le modèle obtenu par la méthode "log-linéaire " surestime la production laitière en début de lactation, lorsque les données ont des valeurs élevées dont le poids est diminué par la transformation logarithmique. En revanche, le modèle obtenu par estimation nonlinéaire des paramètres permet de conserver un poids égal à chaque observation. Pour l'estimation non-linéaire, Соввy \& LE Du (1978) observent que la qualité globale de l'ajustement est meilleure puisque l'écart-type résiduel est plus faible : $2,9 \mathrm{~kg}$ contre $5,8 \mathrm{~kg}$. Cependant l'exemple présenté par ces auteurs indique encore, à notre sens, la possibilité d'un biais en fonction du stade de lactation, avec une sous-estimation de la production en milieu de lactation. L'inconvénient de l'ajustement non-linéaire est qu'il implique d'estimer les paramètres par des méthodes d'approximations numériques itératives, plus coûteuses en temps de calcul.

Une solution intermédiaire employée par CoBby \& LE Du (1978) consiste à 
pondérer les logarithmes des productions laitières observées par un coefficient proportionnel au carré de la valeur observée, puis à appliquer un calcul de régression linéaire classique aux données pondérées. Cette méthode leur a fourni des résultats comparables à ceux de la méthode non-linéaire. Shimizu \& UMrod (1976) avaient déjà utilisé cette méthode de régression linéaire pondérée pour l'estimation des paramètres du modèle de Wood. Mais ils employaient comme coefficient de pondération la production laitière elle-même. SchaEfFEr et al. (1977) ont utilisé quant à eux comme terme de pondération la variance de l'ensemble des valeurs observées à une date donnée pour ajuster la courbe de lactation moyenne du troupeau.

\section{Aptitude du modèle de Woon à s'adapter aux variations de la forme de la courbe de lactation}

Dans plusieurs travaux où le modèle de Wood a servi à ajuster les courbes de production laitière, les auteurs ont cherché à quantifier les effets de différents facteurs de variation de la forme de la courbe de lactation sur les valeurs des paramètres du modèle. Les résultats présentés proviennent d'analyses de variance effectuées sur les valeurs estimées des paramètres; ils sont à considérer avec prudence, étant donné qu'aucun élément dans la bibliographie ne permet de conclure quant à la normalité de la distribution de ces paramètres. Les facteurs de variation de la courbe de lactation les plus souvent étudiés sont le numéro de lactation et la saison de mise-bas, certains auteurs ont également cherché à savoir si les paramètres du modèle de Woon variaient avec le potentiel de production, ou avec la durée de l'intervalle entre vêlages.

- Influence du numéro de lactation (fig. 5a, b, c). Dans toutes les études, l'effet numéro de lactation sur le paramètre a est significatif (WoOD, 1969, 1970 ; KellogG, Urquhart \& Ortega, 1977 ; Killen \& Keane, 1978 ; Madalena, Martinez \& Freitas, 1979 ; Singh, Bhat \& Kumar, 1979 ; Abubakar \& Buvanendran, 1981 ; SChneeberger, 1981 ; Batra, 1986). Comme le montre la figure 5a, celui-ci augmente avec le numéro de lactation, surtout entre la première lactation et les suivantes. Les coefficients b et $\mathrm{c}$ tendent également à augmenter avec le numéro de lactation (WoOD, 1969, 1970, 1980 ; KellogG, Urquhart \& Ortega, 1977 ; Killen \& Keane, 1978 ; Singh, Bhat \& Kumar, 1979 ; Schneeberger, 1981 ; Batra, 1986 ; fig. 5b, c). De telles variations des paramètres permettent des ajustements de qualité satisfaisante, avec des coefficients de corrélation multiple souvent supérieurs à 0,80 (tabl. 2), mais aussi des ajustements de qualité médiocre, surtout dans le cas des premières lactations, pour lesquelles le coefficient de corrélation multiple est en moyenne de l'ordre de 0,60 (Rowlands, LuCEY \& Russel, 1982).

Woov signale également un effet significatif du numéro de lactation sur les valeurs de s, qui diminue quand le rang de lactation augmente (WooD, 1968, 1970) et sur les valeurs de $t_{\max }$, qui sont minimum en $3^{\mathfrak{c}}$ lactation (WoOd, 1968). Ces résultats indiquent que les différences de numéro de lactation contribuent aux corrélations significatives entre les paramètres $a, b$ et $c$ évoquées plus haut.

- Influence de la saison de mise-bas. Bien qu'Abubakar \& Buvanendran (1981) ne trouvent pas d'effet significatif de la saison de mise-bas sur les valeurs des paramètres du modèle de WooD, il ressort des autres travaux que le coefficient a est significativement plus élevé dans le cas de vêlages de printemps ou d'été (Wood, 1968, 1969, 1972, 1976, 1980 ; Killen \& Keane, 1978 ; Madalena, Martinez \& Freitas, 1979 ; Singh, Bhat \& Kumar, 1979 ; Congleton \& Everett, 1980b ; Schneeberger, 1981 ; Batra, 1986). Pour tenir compte de cet effet, Wood (1969) applique, à 
l'équation moyenne obtenue pour l'ensemble d'un troupeau, un facteur multiplicateur dépendant du mois de vêlage, ce qui revient à des estimations différentes du paramètre a selon les mois de mise-bas.

Les paramètres b et $c$ sont eux aussi significativement influencés par la saison de mise-bas, excepté dans l'étude de SchNeEBERGER (1981). Mais les résultats varient avec le site géographique où ils ont été obtenus, et on ne peut en dégager de loi de variation.

- Autres facteurs de variation. D'autres facteurs de variation de la courbe de lactation et des paramètres du modèle de Woop ont été mis en évidence. Ainsi CONGLETon \& Everetr (1980b) soulignent que les paramètres a et b augmentent de manière significative avec le niveau de production totale moyen du troupeau, et avec le niveau de production totale de la vache ou du groupe de vaches au sein d'un troupeau donné, alors que c diminue. Selon ces mêmes auteurs, le paramètre $c$ est également affecté par la durée de l'intervalle mise-bas-insémination fécondante. Pour SchneEberGER (1981), les 3 paramètres varient significativement avec la durée de cet intervalle : plus celui-ci est long, plus a est élevé et plus b et c sont faibles. Selon Batra (1986), seuls les paramètres $\mathrm{b}$ et $\mathrm{c}$, qui sont liés plutôt à la partie décroissante de la courbe, sont significativement influencés par la durée de l'intervalle mise-bas-insémination fécondante.

\section{Modèles utilisés pour décrire les systèmes à compartiments}

Les systèmes à compartiments peuvent être modélisés à l'aide de fonctions du type (ATKINS, 1973) :

$$
F(t)=\sum_{i} a_{i} \cdot e^{-b_{i}} \cdot t
$$

en particulier, l'expression à quatre paramètres :

$$
\operatorname{PL}(t)=a_{1} \cdot e^{-b_{1}} \cdot t-a_{2} \cdot e^{-b_{2}} \cdot t
$$

qui modélise certains types de systèmes à deux compartiments peut être utilisée pour ajuster des courbes de lactation, au même titre que des courbes d'excrétion fécale de marqueurs du transit digestif (Grovum \& Williams, 1973). Cobby \& Le Du (1978) ont proposé ce modèle, sous l'hypothèse d'égalité des paramètres $a_{1}$ et $a_{2}$, en lui donnant une présentation proche du modèle de WooD :

$$
\operatorname{PL}(t)=a \cdot\left(1-\mathrm{e}^{-b \cdot t}\right) \cdot e^{-c \cdot t}
$$

Sous cette forme, l'expression $\left(1-\mathrm{e}^{-\mathrm{b} .1}\right)$ se substitue en effet à la fonction puissance $\mathbf{t}^{\mathrm{b}}$ et permet, selon ces auteurs, de mieux ajuster la phase ascendante de la courbe de lactation que le modèle de WooD, tout en obtenant des paramètres plus indépendants. Nous avons comparé les ajustements obtenus avec les modèles (14) et (19) sur 54 lactations de vaches Frisonnes (tatl. 3). Nous avons trouvé une corrélation significative de $+0,85(\mathrm{P}<0,01)$ entre les paramètres a de ces deux équations. Ces paramètres peuvent tous deux être interprétés comme des estimateurs de la production du début de la lactation, et en particulier de la production maximale $\mathrm{PL}_{\max }$ (fig. 6), à laquelle ils sont fortement corrélés : $+0,94$ et $+0,86(\mathbf{P}<0,01)$ respectivement. Dans ce travail, les paramètres $b$ des équations de Woon et de CoBBy \& LE Du sont inversement et significativement corrélés : $-0,57 \cdot(\mathrm{P}<0,01)$; par contre, les paramètres c des deux modèles sont indépendants. 
Globalement, l'équation (19) n'est pas un meilleur modèle que l'équation (14). En effet, les travaux de Rowlands, LuCEY \& Russel (1982) et les nôtres montrent que les écarts-types résiduels sont du même ordre de grandeur dans les deux cas, et que les deux modèles expliquent la même part de variation de la production (tabl. 2). Cependant, les comparaisons d'ajustement que nous avons obtenues sur les 20 premières semaines de la lactation, indiquent que le modèle (19) s'adapte mieux aux courbes présentant un accroissement important de la production en début de lactation.

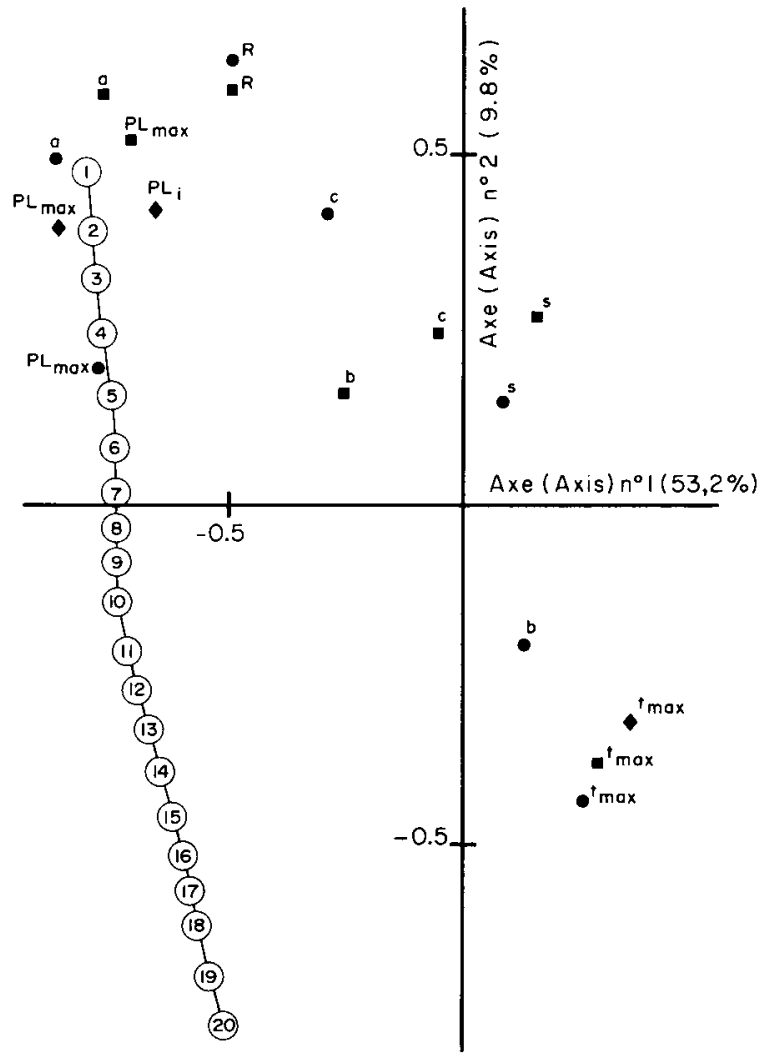

- Valeurs observées.

Observed values.

- Valeurs estimées par le modèle de Woon.

Wood's model estimations.

- Valeurs estimées par le modèle de CobBy \& LE Du.

COBBY \& LE Du's model estimations.

FIG. 6

Analyse en composantes principales de 54 courbes de lactation de vaches Frisonnes: corrélations entre les paramètres du modèle de WooD, ceux du modèle de COBBY \& LE DU, et différentes caractéristiques de la courbe de lactation.

Principal component analysis of 54 Friesian cow lactation curves : correlations between Woon's model parameters, СоввY \& LE DU's model parameters and different characteristics of the curve. 
Un modèle proche de l'équation (19) a été utilisé par Minder \& McMillan (1977) qui proposent d'ajuster la production d'œufs par l'équation :

$$
N(t)=a \cdot e^{-c \cdot t} \cdot\left(1-e^{-b \cdot t}\right) / b
$$

avec $\mathrm{N}(\mathrm{t})$ : nombre d'œufs produits le jour $\mathrm{t}$

$\mathrm{a}, \mathrm{b}, \mathrm{c}$ : paramètres du modèle.

En outre, SchaEfFer et al. (1977) ont appliqué ce modèle à la production laitière, avec l'introduction d'un quatrième paramètre, $t_{0}$, qui s'interprète comme la date théorique à laquelle la mamelle commence à produire du lait avant la mise-bas :

$$
\text { PL }(t)=\text { a.e. }^{-c \cdot\left(t-t_{0}\right)} \cdot\left(1-e^{-b \cdot\left(1-t_{0}\right)} / b\right.
$$

Un modèle du même type a également été utilisé pour la production d'œufs par Gavaro, Parker \& McMillan (1971) et par Gavora et al. (1982), modèle proposé par McMillan, Fitz-Earle \& Robson (1970) et par McMillan et al. (1970) :

$$
N(t)=a \cdot e^{-b \cdot t} \cdot\left(1-e^{-c \cdot\left(t-t_{0}\right)}\right)
$$

La présence du paramètre $t_{o}$ dans les équations (21) et (22) permet d'obtenir une estimation non nulle de la production initiale. La prise en compte d'une production initiale non nulle pourrait également être effectuée en admettant des valeurs différentes des paramètres $a_{1}$ et $a_{2}$ dans l'équation (18). La différence $\left(a_{1}-a_{2}\right)$ est alors une estimation de la production initiale.

Le calcul des paramètres des modèles (19) à (22) implique l'usage de méthodes de régression non-linéaire.

\section{Autres modèles non-linéaires}

\section{Modèles dérivés du modèle de WooD} WOOD :

Dhanoa (1981), a proposé une écriture légèrement différente du modèle de

$$
\text { PL }(\mathrm{t})=\mathrm{a} \cdot \mathrm{t}^{\mathrm{b} \cdot \mathrm{c}} \cdot \mathrm{e}^{-\mathrm{c} \cdot \mathrm{t}}
$$

Une telle équation permet de réduire les corrélations entre paramètres dans de nombreux cas. Notamment, dans 10 cas sur les 11 qu'il a étudiés, la corrélation entre les paramètres $b$ et $c$ est devenue inférieure à 0,8 , valeur qui reste cependant assez élevée. De plus, ce modèle fournit, grâce au paramètre $b$, une estimation directe de la date du pic de lactation (tabl. 1).

Partant d'une étude de Wood (1969) mettant en évidence un effet «mise à l'herbe » sur la production laitière, Kuck, Grossman \& NorTon (1976) proposent de corriger le niveau de production estimé à l'aide de la fonction Gamma (14) par un facteur multiplicateur, qui est fonction du jour de l'année pour lequel on calcule PL ( $t$ ). L'équation s'écrit alors :

$$
\mathrm{PL}_{\mathrm{x}}(\mathrm{t})=\mathrm{a} \cdot \mathrm{t}^{\mathrm{b}} \cdot \mathrm{e}^{-\mathrm{c} \cdot \mathrm{t}} \cdot(1+\mathrm{u} \cdot \cos (\mathrm{x})+\mathrm{v} \cdot \sin (\mathrm{x}))
$$

avec $\operatorname{PL}_{\mathrm{x}}(\mathrm{t})$ : production laitière estimée pour le t-ième jour de lactation et le $\mathrm{x}$-ième jour de l'année

a. $t^{b} \cdot e^{-c . t}:$ modèle de Woon (équation 14 )

$\mathrm{u}, \mathrm{v}$ : paramètres

$\cos (\mathrm{x}), \sin (\mathrm{x}): \operatorname{cosinus}$ et sinus de $\mathrm{x}$, exprimé en radians. 
Ce modèle assez séduisant nécessite une linéarisation par transformation logarithmique pour l'estimation des 5 paramètres $\mathrm{a}, \mathrm{b}, \mathrm{c}$, u et $\mathrm{v}$ :

$$
\text { Ln } P L_{x}(t)=\operatorname{Ln} a+b \cdot \operatorname{Ln} t-c \cdot t+u \cdot \cos (x)+v \cdot \sin (x)
$$

puisque pour $-1<\mathrm{u} \cdot \cos (\mathrm{x})+\mathrm{v} \cdot \sin (\mathrm{x})<1$, on peut faire l'approximation suivante :

$$
\operatorname{Ln}(1+u \cdot \cos (x)+v \cdot \sin (x))=u \cdot \cos (x)+v \cdot \sin (x)
$$

La méthode de régression linéaire multiple est alors applicable. Une analyse de variance effectuée sur les valeurs des paramètres montre que les coefficients u et $v$ varient significativement avec le mois de mise-bas $(P<0,01)$, mais les résultats publiés ne permettent pas d'en préciser les sens de variation (Grossman, Kuck \& Norton, 1986).

Sauvant \& Fehr (1976) utilisent, pour ajuster les courbes de lactation des chèvres, le modèle de WooD, auquel ils ajoutent une constante :

$$
\operatorname{PL}(\mathrm{t})=\mathrm{a}+\mathrm{b} \cdot \mathrm{t}^{\mathrm{c}} \cdot \mathrm{e}^{\mathrm{d} \cdot \mathrm{t}}
$$
initiale.

Dans cette équation, le terme a apparaît comme un estimateur de la production

De même, Schneeberger (1981), trouvant que le modèle de Wood s'ajuste parfois mal aux productions du début de la lactation, introduit un quatrième paramètre, $t_{0}$, présentant la signification évoquée pour l'équation (21) :

$$
\operatorname{PL}(\mathrm{t})=\mathrm{a} \cdot\left(\mathrm{t}-\mathrm{t}_{\mathrm{o}}\right)^{\mathrm{h}} \cdot \mathrm{e}^{-\mathrm{c} \cdot\left(\mathrm{t}-\mathrm{t}_{\mathrm{t}}\right)}
$$

Cette expression présente l'avantage, par rapport à celle de Wood, de tenir compte du fait que la production laitière initiale effective n'est pas nulle. Une telle écriture permet en outre de réduire la fréquence des cas où l'estimation de b est négative, ce qui signifie que la courbe ne présente pas de maximum de production.

MCNally (1971) a ajusté la production d'œufs des poules pondeuses, $N(t)$, selon l'équation :

$$
N(t)=a \cdot t^{h} \cdot e^{-c \cdot t+d \cdot t^{1 / 2}}
$$

Ce modèle est à rapprocher de l'exponentielle parabolique proposée par SikKa (1950). En effet, on y retrouve le fait que la décroissance de la production est globalement exponentielle, selon un rythme moyen défini par le paramètre $c$, mais avec des variations locales par rapport à ce rythme moyen définies par le paramètre $\mathrm{d}$.

Enfin, citons Vujicic \& Bacic (1961) qui proposent le modèle :

$$
\operatorname{PL}(\mathrm{t})=\mathrm{t} \cdot \mathrm{a}^{-\mathrm{b}} \cdot \mathrm{e}^{-\mathrm{a} \cdot \mathrm{t}}
$$

Cette expression est voisine de celle proposée par Wood, mais son inconvénient est qu'elle nécessite pour chacun des paramètres des estimations différentes pour chaque mois de lactation.

Pour décrire les courbes de lactation des vaches laitières, Jenkins \& Ferrell (1982) proposent le modèle :

$$
\operatorname{PL}(\mathrm{t})=\mathrm{t} /\left(\mathrm{a} \cdot \mathrm{e}^{\mathrm{k} \cdot \mathrm{t}}\right)
$$

qu'ils appliquent également à la modélisation de courbes de lactation de vaches allaitantes de race à viande (Jenkins \& Ferrell, 1984). Si l'on modifie l'écriture de ce modèle de la manière suivante :

$$
\text { PL }(t)=a^{-1} \cdot t \cdot e^{-k \cdot t}
$$

on retrouve le modèle de Woon dans le cas particulier où b égale 1 . 


\section{Modèles non-linéaires ne s'inspirant pas du modèle de WooD}

Quelques autres modèles figurent dans la bibliographie et peuvent s'appliquer à la courbe de lactation. Ainsi, outre l'équation (19), CoBby \& LE Du (1978) proposent le modèle :

$$
\operatorname{PL}(\mathrm{t})=\mathrm{a} \cdot\left(1-\mathrm{e}^{\mathrm{b} \cdot \mathrm{t}}\right)-\mathrm{c.t}
$$

Selon ces auteurs, les modèles (19) et (30) sont à employer selon que la courbe présenté unè décroissançe se rapprochant asymptotiquement plutôt d'une exponentielle ou plutôt d'une droite", respectivement.

OstergaARD (1979) ajuste l'évolution de la quantité de matière sèche ingérée (MSI) par les vaches laitières, qui présente une allure générale comparable à la production laitière brute, selon l'équation :

$$
\operatorname{MSI}(\mathrm{t})=\mathrm{a}-\mathrm{b} \cdot \mathrm{e}^{-\mathrm{c} \cdot \mathrm{t}}+\mathrm{d} . \mathrm{t}
$$

En cas d'égalité des paramètres a et $b$, cette équation se ramène au modèle (28) proposé par CoBBY \& LE Du (1978).

Neloer (1966) propose une famille de fonctions polynômiales inverses pour ajuster les courbes de réponse dans les expériences plurifactorielles. L'auteur démontre l'intérêt de ces fonctions à propos des courbes de variation des rendements de céréales à différents niveaux de fertilisation et l'une d'elles est employée dans les travaux de modélisation de la courbe de lactation par Singh et Bhat (1978), Singh, Bhat \& Kumar (1979), Bhat, Kumar \& Garg (1981) et Batra (1986) :

$$
\operatorname{PL}(\mathrm{t})=\mathrm{t} /\left(\mathrm{a}+\mathrm{b} \cdot \mathrm{t}+\mathrm{c} \cdot \mathrm{t}^{2}\right)
$$

BATRA (1986) a montré que les paramètres a et c sont significativement plus faibles dans le cas des premières lactations $(P<0,01)$, et que les trois paramètres varient significativement avec le mois de mise-bas et avec la durée de l'intervalle tmise-basinsémination fécondante $(\mathrm{P}<0,05)$.

\section{Modèles prenant en compte le phénomène d'autocorrélation}

Tous les modèles exposés jusqu'à présent sont de type déterministe. Dès lors que le stade de lactation est fixé, la production laitière est coṇsidérée comme cónnue, aux erreurs d'estimation près.

Une autre approche de la modélisation de la production laitière consite à tenir compte du phénomène d'autocorrélation. En effet, lè niveau de production à un stade de lactation t est largement déterminé par le niveau de production observé au stade de lactation $(t-1)$. Ainsi, au cours des 20 premières semaines de lactation de 54 vaches Frisonnes, nous avons observé que les productions laitières de deux contrôles hebdomadaires successifs présentent un coefficient de corrélation toujours significatif, au moins égal à 0,8 . Ce phénomène est en outre à l'origine du facteur de taille qui est systématiquement extrait par une analyse en composantes principales appliquée à des courbes de lactation (fig. 2). L'autocorrélation intervient également par le fait qu'un écart brutal à la trajectoire normale ne se résorbe, quelles que soient les interventions effectuées, que d'une façon lente et souvent incomplète. 
Dans les 2 exemples qui suivent, l'allure générale de la courbe est fournie par les paramètres d'un modèle déterministe et les valeurs des productions observées sont obtenues par l'intégration d'une variation de part et d'autre de la tendance.

\section{A. Le modèle de DHANOA \& LE DU}

Dhanoa \& Le Du (1982) ont construit leur modèle en 2 étapes. D'abord, ils ont choisi un modèle déterministe d'ajustement de la courbe de lactation : pour des raisons de simplicité, ils ont retenu le modèle linéaire du premier degré. Ensuite, partant d'une observation de TURNER (1926) selon laquelle, dans des conditions normales, la production laitière moyenne mensuelle est, en phase décroissante de la lactation, une fraction constante de la production moyenne du mois précédent, DhanoA \& Le Du (1982) ont fait l'hypothèse que la variation de production observée entre 2 semaines de lactation consécutives, $\left(\mathrm{PL}_{\mathrm{o}}(\mathrm{t})-\mathrm{PL}_{\mathrm{o}}(\mathrm{t}-1)\right)$, était proportionnelle à la variation de production espérée, $\left(\mathrm{PL}_{\mathrm{e}}(\mathrm{t})-\mathrm{PL}_{\mathrm{o}}(\mathrm{t}-1)\right)$, connaissant le niveau de production en semaine $(t-1)$ :

$$
\mathrm{PL}_{\mathrm{o}}(\mathrm{t})-\mathrm{PL}_{\mathrm{o}}(\mathrm{t}-1)=\mathrm{k} \cdot\left(\mathrm{PL}_{\mathrm{c}}(\mathrm{t})-\mathrm{PL}_{\mathrm{o}}(\mathrm{t}-1)\right)
$$

d'où :

$$
\mathrm{PL}_{\mathrm{o}}(\mathrm{t})=k \cdot \mathrm{PL}_{\mathrm{e}}(\mathrm{t})+(1-\mathrm{k}) \cdot \mathrm{PL}_{\mathrm{o}}(\mathrm{t}-1)
$$

avec $\mathrm{PL}_{\mathrm{o}}(t-1)$ : production observée en semaine $(t-1)$

$\mathrm{PL}_{\mathrm{o}}(\mathrm{t})$ : production observée en semaine $t$

$\mathrm{PL}_{e}(\mathrm{t})$ : production de la semaine $\mathrm{t}$, estimée à partir du modèle linéaire

$\mathrm{k}$ : scalaire compris entre 0 et 1 .

Le scalaire $\mathrm{k}$ représente donc le rapport, supposé constant tout au long de la lactation, entre les variations de production observée et de production estimée entre deux stades de lactation consécutifs. Par suite, $\mathbf{k}$ paraît déterminer le degré de priorité de la tendance générale décrite par le modèle déterministe. Cependant, selon une étude de Hoekstra (1986) portant sur le modèle (33) où $\mathrm{PL}_{\mathrm{e}}(\mathrm{t})$ suit le modèle linéaire simple, plus $\mathrm{k}$ est voisin de 1 , plus le maximum de production estimé intervient tôt et plus la courbe de lactation estimée se rapproche rapidement d'une droite. L'auteur conclut alors que le paramètre $\mathrm{k}$ est en partie déterminé par l'intervalle séparant la mise-bas du pic de production, et non pas par la corrélation entre les niveaux de production successifs.

Un tel modèle permet de rendre compte des variations à court terme de la production laitière, liées à des variations de conditions environnementales et/ou expérimentales (fig. 7c), ce que ne font pas les modèles déterministes présentés dans les précédents paragraphes (fig. $7 \mathrm{a}, \mathrm{b}$ ).

\section{B. Le modèle de Goodall \& SprevaK}

L'ajustement d'une courbe de lactation par un modèle permet de prévoir la production laitière $\mathrm{PL}_{\mathrm{e}}(\mathrm{t})$ à un stade $\mathrm{t}$ de lactation, avec une erreur $\epsilon(t)$ par rapport à la production réelle observée $\mathrm{PL}_{\mathrm{o}}(\mathrm{t})$, de telle sorte que le modèle s'écrit :

$$
\mathrm{PL}_{\mathrm{o}}(\mathrm{t})=\mathrm{PL}_{\mathrm{e}}(\mathrm{t})+\epsilon(\mathrm{t})
$$

Mais Goodall \& SPREvaK (1984) préfèrent une écriture où le terme " erreur » $E(t)$ figure comme facteur multiplicateur de la production estimée par un modèle déterministe :

$$
\mathrm{PL}_{\mathrm{o}}(\mathrm{t})=\mathrm{PL}_{\mathrm{e}}(\mathrm{t}) \cdot \mathrm{E}(\mathrm{t})
$$




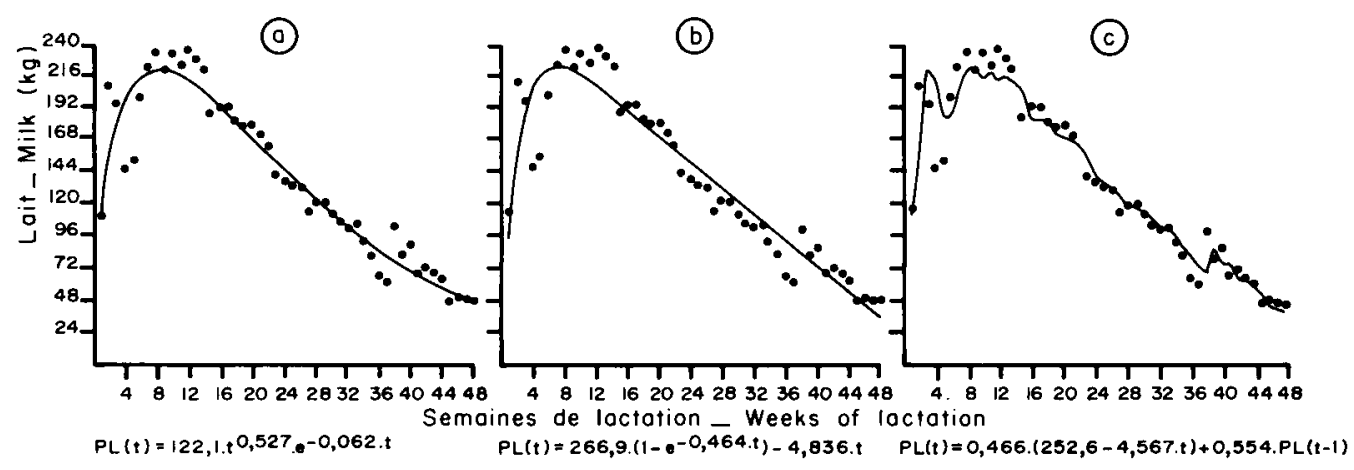

FIG. 7

Ajustement de la courbe de lactation d'une vache laitière par les modèles de WooD (a), de Cobry \& Le Du (b) et par le modèle de Dhanoa \& Le Du (c) (Dhanoa \& Le Du, 1978). Adjustment of a dairy cow lactation curve by Wood's model (a), or COBBY \& LE DU's (b), or DHanoa \& LE Du's (c).

ce qui leur permet une linéarisation complète du modèle :

$$
\text { Ln } \mathrm{PL}_{\imath}(\mathrm{t})=\mathrm{Ln} \mathrm{PL}_{\mathrm{e}}(\mathrm{T})+\mathrm{Ln} \mathrm{E}(\mathrm{t})
$$

Goodall \& SprevaK (1984) montrent que la série des termes Ln E(t) est hautement autocorrélée durant la lactation, et proposent de traduire cette autocorrélation par une relation linéaire du type :

$$
\operatorname{Ln} E(t)=k \cdot \operatorname{Ln} E(t-1)+E^{\prime}(t)
$$

où $E^{\prime}(t)$ représente une erreur qui suit une loi normale de moyenne nulle. Le scalaire $k$ est, en valeur absolue, strictement compris entre 0 et 1 , et est estimé par régression linéaire sur l'ensemble des lactations d'un troupeau.

De cela, ils déduisent un modèle stochastique de description de la courbe de lactation :

$$
\mathrm{PL}_{\mathrm{o}}(\mathrm{t})=\left[\frac{\mathrm{PL}_{\mathrm{o}}(\mathrm{t}-1)}{\mathrm{PL}_{\mathrm{e}}(\mathrm{t}-1)}\right]^{\mathrm{k}} \cdot \mathrm{PL}_{\mathrm{e}}(\mathrm{t})
$$

Comme dans le cas du modèle de Dhanoa \& Le Du (1982), le modèle rend mieux compte des variations à court terme de production laitière autour d'une allure générale de courbe, définie par les paramètres du modèle déterministe employé.

Dans un article plus récent (Goodall \& Sprevak, 1985) les auteurs présentent la méthode récursive dite du filtre de Kalman (Kalman, 1960, 1963 ; Kalman \& Bucy, 1961) qui permet simultanément l'estimation du paramètre $k$ et celle des paramètres du modèle déterministe employé pour prévoir $\mathrm{PL}_{\mathrm{e}}(\mathrm{t})$ dans l'équation (37). De plus, ils montrent comment un tel modèle permet une estimation précoce de la courbe de lactation d'une vache laitière. En effet, avec un modèle déterministe, dont on estime les paramètres par la méthode des moindres carrés, il faut disposer des données de production laitière des 15 premières semaines au moins pour avoir une estimation de la production totale aussi satisfaisante que celle obtenue en appliquant les valeurs moyennes des paramètres calculées sur les précédentes lactations du troupeau. En revanche, la combinaison de la méthode du filtre de Kalman et du modèle (37) permet 
de réduire à 10 , voire 5 dans certains cas, le nombre minimum d'observations hebdomadaires nécessaires à l'estimation de la courbe. Ainsi, avec les résultats de 10 semaines de lactation pour un troupeau de 92 vaches, les auteurs font une erreur moyenne de $6 \%$ seulement dans la prédiction de la quantité totale de lait produite.

\section{Discussion et conclusions}

Il ressort de cette étude que, malgré le large éventail des modèles disponibles pour ajuster les courbes de lactation, la situation ne peut pas être considérée comme satisfaisante en raison de l'importance des remarques qui peuvent être adressées à la plupart des démarches et des modèles proposés. Le principal facteur d'émergence des modèles d'ajustement a été jusqu'alors la facilité de calcul des paramètres. A ce propos, une critique quasi générale des différentes propositions peut être faite sur l'insuffisance de l'évaluation de la qualité statistique des ajustements. En effet le coefficient de détermination, qui ne constitue qu'un élément très partiel de cette évaluation, est le plus souvent le seul paramètre utilisé à cette fin. La variation résiduelle n'est pas toujours calculée et sa structure n'a quasiment jamais été étudiée. Enfin les erreurs sur les coefficients de régression estimés, ainsi que les corrélations entre paramètres n'ont pas été considérées.

Le caractère ajusté demeure dans la plupart des cas la production laitière brute, or il semblerait logique de chercher à modéliser les niveaux de sécrétion des principaux constituants du lait: lactose, protéines, lipides et matières minérales. En effet ces caractères se rapprochent plus, d'une part, de la valeur «beurrière " ou " fromagère », voire "économique " de la production et, d'autre part, de l'intensité des phénomènes biologiques associés à la sécrétion lactée.

Il est regrettable que d'autres aspects importants de la courbe de lactation n'aient pour ainsi dire pas été jusqu'à présent l'objet d'études spécifiques. Ainsi les variations à court terme, par exemple d'un jour à l'autre, de la production laitière méritent notre attention. En effet, grâce aux progrès des méthodes de saisie, ces informations sont actuellement déjà enregistrées dans un certain nombre d'élevages. Il importe donc de pouvoir les interpréter et, en particulier, d'élaborer une démarche permettant de détecter les variations anormales. D'autre part, à partir du moment où, sous l'influence d'un facteur exogène, la production s'est éloignée significativement de sa trajectoire la plus probable, il importerait d'étudier la ou les lois du retour à une trajectoire normale. Dans ce domaine il semble indispensable de développer la voie tracée par Dhanoa \& LE Du (1982) et Goodall \& Sprevak (1984) pour aboutir à des modèles qui soient capables d'intégrer l'existence des variations de la production autour d'une trajectoire moyenne. De tels modèles pourraient également être utilement appliqués dans le cadre de la valorisation des résultats du contrôle laitier. En effet, la règle actuellement adoptée consiste à souligner une chute mensuelle de production supérieure à $10 \%$, en ne considérant que deux enregistrements consécutifs. Or l'application de modèles stochastiques devrait permettre d'aboutir à une meilleure interprétation des variations de production mensuelles en intégrant plus largement le passé de chaque courbe de lactation.

En admettant que le niveau de production laitière d'un animal à un moment donné est déterminé par celui de son facteur biologique le plus limitant, il apparaît que les 
études considérées dans ce travail n'ont pas cherché à identifier ce facteur et à évaluer son influence sur la production. Or il est probable que les valeurs calculées des paramètres varient sensiblement suivant, par exemple, que la courbe considérée se rapproche d'un idéal ou bien traduit des niveaux de disponibilité d'un ou plusieurs facteurs limitants. Il semble que les études publiées jusqu'alors sur les modèles d'ajustement de la production laitière, ont plutôt concerné des courbes moyennes et assez proches d'un idéal, les modèles empiriques ainsi obtenus ne sont vraisemblablement pas capables de s'adapter à la variété des courbes observées en pratique puisqu'ils n'ont pas été conçus dans ce but. Il paraît possible de progresser assez rapidement dans ce domaine : en effet, de très nombreux travaux ont concerné l'influence du niveau d'un facteur alimentaire, sur une partie au moins de la courbe de lactation. Une interprétation globale de ces résultats regroupés en une base de données devrait permettre la mise au point de modèles capables de s'adapter à l'éventail des situations alimentaires rencontrées en pratique. De tels modèles seraient en outre de précieux outils pour hiérarchiser des priorités de recherche et pour accroître la pertinence de certaines démarches de développement.

Un avantage essentiel du modèle linéaire du premier degré est sa simplicité, ainsi que la facilité de l'estimation et de l'interprétation des paramètres. Cependant, le principal défaut des expressions linéaires simples résides dans leur incapacité à ajuster simultanément de manière satisfaisante les deux phases d'une courbe de production laitière. Pour les modèles polynômiaux, la linéarité vis-à-vis de leurs paramètres leur confère un avantage de simplicité d'estimation par la méthode des moindres carrés. En revanche, de telles équations ne peuvent servir à décrire la production de lait avec des paramètres systématiquement interprétables d'un point de vue biologique ou zootechnique. D'une part, les modèles polynômiaux de degré supérieur à 2 possèdent au moins deux extrema. Dans le cas d'un modèle de courbe de lactation, si la présence d'un maximum est souhaitable, l'existence d'un minimum au-delà duquel la fonction est de nouveau croissante n'a pas de sens. Ceci limite l'emploi de ces modèles pour la prévision des productions laitières d'animaux présentant des lactations particulièrement longues.

Les modèles proposés ci-dessus ne s'appliquent valablement qu'à certaines souspériodes de la lactation. Les équations correspondantes sont éventuellement intéressantes pour étudier l'évolution des différences de production observées par exemple sous l'effet d'un traitement par rapport à un régime témoin.

La fonction Gamma, proposée par Wood (1967), est devenue le modèle de référence pour l'ajustement des courbes de lactation. Ceci s'explique, d'une part, par la qualité des ajustements obtenus dans la plupart des cas, et d'autre part, par le fait que l'estimation des paramètres reste simple puisque le modèle est linéarisable. De plus, cette équation possède un nombre restreint de paramètres, qui peuvent être rapprochés de différentes périodes de la courbe de lactation : b pour la phase ascendante, a pour le maximum de production et $c$ pour la phase décroissante. L'application de cette fonction n'est cependant pas exempte de critiques importantes. En particulier, les paramètres ne sont pas indépendants et aucun d'eux ne peut être interprété de façon simple au regard des caractéristiques classiques de la courbe de lactation. Enfin d'après nos observations, la fonction Gamma ne peut s'appliquer sans discernement à des courbes de lactation individuelles. En effet, une proportion non négligeable d'animaux apparemment sans problème présentent des formes de courbes qui ne peuvent être ajustées correctement par ce modèle. Il convient donc de nuancer l'unanimité que le modèle de Wood semble faire dans la littérature. D'ailleurs, certaines variantes propo- 
sées de ce modèle sont parfois très intéressantes pour affiner l'ajustement ou la prévision de la production laitière.

Wood (1977) a tenté de fournir, a posteriori, une explication biologique des paramètres de son équation. Cependant, les mécanismes évoqués paraissent être en partie contestables. Quelques auteurs ont tenté de modéliser les principaux processus digestifs et/ou métaboliques des ruminants laitiers. Certains modèles proposés présentent cependant l'inconvénient de considérer l'équation de Woon comme une donnée du problème (BRUCE et al., 1984 ; Forbes, 1977 ; WoOD, 1979) tandis que d'autres n'intègrent pas la possibilité de moduler la production laitière en fonction des valeurs des caractéristiques métaboliques modélisées (BYwATER, 1984). Le modèle explicatif le plus avancé est vraisemblablement celui de NEAL \& THORNLEY (1983), lequel intègre les effets de 3 facteurs : un flux d'apport de nutriments, une sécrétion d'hormone et le nombre de cellules sécrétrices. Même s'il est criticable en raison de la simplification, à notre sens excessive, des phénomènes nutritionnels et hormonaux sous-jacents, ce modèle explicatif ouvre certainement la voie à la mise au point d'autres propositions plus fidèles au fonctionnement de l'organisme d'un ruminant laitier. Il serait à ce propos très souhaitable de privilégier l'usage de paramètres qui seraient facilement interprétables en fonction des caractéristiques classiques de la courbe de lactation et de l'état physiologique ou nutritionnel de l'organisme.

La limite des possibilités de calcul a indéniablement freiné la mise au point de modèles plus satisfaisants. Il semble que le développement de l'informatique et de logiciels adaptés devrait permettre de faire évoluer sensiblement la situation présente. Toutefois, le risque d'aboutir ainsi à un modèle lourd et complexe n'est pas négligeable, ce qui signifie qu'il ne faut pas délaisser la mise au point d'une gamme de modèles simples et opérationnels adaptés aux objectifs précis des différents utilisateurs (alimentation, génétique...).

Reçu en mai 1986. Accepté en février 1987.

\section{Summary \\ Adjustment models for the lactation curves}

Though milk production always follows the same general trend (fig. 1), statistical description of milk production records shows that lactation curves can be ranked according to their shape, defined by the relative importance of milk yields in the early stages of lactation and milk yields in late lactation, and to the total milk production level (fig. 2, 3). Hence, studying the lactation curve of ruminant females is of great significance as shown by the large number of mathematical models which have been developed to describe or predict it.

Most models of the literature are deterministic ones, whenever linear or not. They allow the calculation of some characteristics of the curve, such as peak yield value and time, persistency after peak yield (tabl. 1) and also total milk production. However, it is often uneasy to find simple relations between the model parameters and those characteristics.

Among deterministic models, Wood's equation (1967), PL $(t)=a \cdot t^{b} \cdot e^{-c . t}$, based on a Gamma function, is the most frequently used. This equation takes into account the rising phase of the curve and it can be linearized for the estimation of the parameters, both reasons explaining its succes. Nevertheless, linearization of WooD's equation by a logarithmic transformation may lead to biased estimation of the parameters and result in an ill-fitting of the lactation curve (fig. 5). Moreover, only parameter a has some significance concerning performance, as a scaling factor highly correlated with milk yiclds at the onset of lactation (fig. 4), and parameters b and $\mathrm{c}$ are shape factors with no precise meaning. Many authors study the factors of variation of the lactation curve by interpreting analysis of variance applied to the parameters of Woon's equation. 
In most situations, satisfactory fits of the general trend of the curve have been obtained with deterministic models (tabl. 2), despite the high correlations observed within models between parameters (tabl. 3). So they are for instance helpful to interpret experimental data. Nevertheless such models are unable to take into account short-term deviations of the lactation curve from its general trend.

Partly for this reason, authors attempted to build up stochastic models, integrating a deterministic component to describe the general shape of the curve, and a function accounting for autocorrelation in milk production and allowing local variations in milk yield (fig. 6, 7). The development of such models is of some interest for analysing milk production records.

Furthermore, it must be noticed that the equations suggested to describe and/or predict milk yield are generally empirical and only seldom attempts have been made to take into account nutritional and physiological events underlying milk secretion. Progress may be achieved in this way with research on modelling of biological systems, with the help of computers.

Key words : lactation, curve, model, estimation, prediction.

\section{Références bibliographiques}

Abubakar B.Y., Buvanendran V., 1981. Lactation curves of Friesian-Bunaji crosses in Nigeria. Livest. Prod. Sci., 8, 11-19.

De Assis A.G., France J., 1983. Modelling dairy cattle feeding in the South-East region of Brazil. Agric. Syst., 12, 129-170.

AtkıNs G.L., 1973. Modèles à compartiments multiples pour les systèmes biologiques. Ed. Gauthier-Villars. Paris, Bruxelles, Montréal, 181 p.

Auran T., Mocquot J.C., 1974. Etudes sur la production laitière des bovins. II. Intérêt des lactations partielles pour la sélection. a) Etude critique de différentes méthodes d'estimation des productions totales à partir de résultats de lactation partielle. Ann. Génét. Sél. anim., 6, 429-444.

Batra T.R., 1986. Comparison of two mathematical models in fitting lactation curves for pureline and crossline dairy cows. Can. J. Anim. Sci., 66, 405-414.

Bhat P.N., Kumar R., Garg R.C., 1981. Note on comparative efficiency of various lactation curve functions in Hariana cattle. Indian J. anim. Sci., 51, 102.

BonNIER G., 1935. Is the shape of the lactation curve genetically determined ? Hereditas, 20, 199. Cité par Delage, Leroy \& Poly, 1953.

Brody S., Ragsdale A.C., Turner C.W., 1923. The rate of decline of milk production with the advance of the period of lactation. J. gen. Physiol., 5, 441. Cités par Delage, Leroy \& Poly, 1953.

Brody S., Turner C.W., Ragsdale A.C., 1924. The relation between the initial rise and subsequent decline of milk secretion following parturition. J. gen. Physiol., 6, 541. Cités par Delage, Leroy \& Poly, 1953.

Bruce J.M., Broadbent P.J., Topps J.H., 1984. A model of the energy system of lactating and pregnant cows. Anim. Prod., 38, 351-362.

Bywater A.C., 1984. A generalised model of feed intake and digestion in lactating cows. Agric. Syst., 13, 167-186.

Cobry J.M., Le Du Y.L.P., 1978. On fitting curves to lactation data. Anim. Prod., 26, 127-133.

Congleton W.R. jr, 1984. Dynamic model for combined simulation of dairy management strategies. J. Dairy Sci., 67, 644-660.

Congleton W.R. jr, Everett R.W., 1980a. Error and bias in using the incomplete Gamma function to describe lactation curves. J. Dairy Sci., 63, 101-108.

Congleton W.R. jr, Everett R.W., 1980b. Application of the incomplete Gamma function to predict cumulative milk production. J. Dairy Sci., 63, 109-119.

Decaen C., Journet M., Poutous M., 1970. Evolution de la production laitière de la vache au cours des deux premiers mois de la lactation. I. Description graphique de l'évolution journalière de la quantité de lait sécrétée, du taux butyreux et de la quantité de matières grasses sécrétée. Ann. Zootech., 19, 191-203. 
Decaen C., Calomiti S., Poutous M., 1970. Evolution de la production laitière de la vache au cours des deux premiers mois de la lactation. II. Analyse de la variation de la quantité de lait. Ann. Zootech., 19, 205-221.

Delage J., Leroy A.M., Poly J., 1953. Une étude sur les courbes de lactation. Ann. Zootech., 3, 225-267.

Dhanoa M.S., 1981. A note on an alternative form of the lactation model of Wood. Anim. Prod., 32, 349-351.

Dhanoa M.S., Le Du Y.L.P., 1982. A partial adjustment model to describe the lactation curve of a dairy cow. Anim. Prod., 34, 243-247.

Draper N., Smith H., 1981. Chap. 10 « An introduction to nonlinear estimation ». 458-529, in : Applied regression analysis, $2^{\mathrm{e}}$ éd. Ed. J. Wiley \& sons Inc. New-York.

EDwardS J., 1950. Factors influencing the relationship between the secretion of milk and butterfat. J. Agric. Sci., Camb., 40, 100. Cité par Delage, Leroy \& Poly, 1953.

FisCHer A., 1958. Untersuchungen an württembergischen Fleckviehkühen über die Form der Laktationskurve und deren Beeinflussung durch nichterbliche Faktoren. Züchtungskunde, 30, 296-304. Cité par Gravert \& Baptist, 1976.

Forbes J.M., 1977. Development of a model of voluntary food intake and energy balance in lactating cows. Anim. Prod., 24, 203-214.

Gaines W.L., 1926. Interpretation of the lactation curve. J. Gen. Physiol., 9, 325. Cité par Delage, Leroy \& Poly, 1953.

Gaines W.L., 1927a. Persistency of lactation in dairy cows. Illinois Agr. Exp. Sta. Bull., 288, 355. Cité par Delage. Leroy \& Poly, 1953.

Gaines W.L., 1927b. Measures of persistency of lactation. J. Agric. Res., 34, 373. Cité par Delage, Leroy \& Poly, 1953.

Gaines W.L., Davidson F.A., 1926. The effect of advance in lactation and gestation on mammary activity. J. Gen. Physiol., 9, 325. Cité par Delage, Leroy \& Poly, 1953.

Gavora J.S., Parker R.J., McMillan I., 1971. Mathematical model of egg production. Poult. Sci., 50, 1306-1315.

Gavora J.S., Liljedahl L.E., McMillan I., Ahlen K., 1982. Comparison of three mathematical models of egg production. Br. Poult. Sci., 23, 339-348.

Gooch M., 1935. An analysis on the time change in milk production in individual lactations. $J$. Agric. Sci., Camb., 25, 71. Cité par Delage, Leroy \& Poly, 1953.

Goodall E.A., Sprevak D., 1984. A note on a stochastic model to describe the milk yield of a dairy cow. Anim. Prod., 38, 133-136.

Goodall E.A., SpREvaK D., 1985. A Bayesian estimation of the lactation curve of a dairy cow. Anim. Prod., 40, 189-193.

Gravert H.O., Baptist R., 1973. Soll man Kühe mit besseren Laktationskurven züchten? Züchtungskunde, 45, 13-21.

Gravert H.O., Baptist R., 1976. Breeding for persistency of milk yield. Livest. Prod. Sci., 3, 27 31 .

Grossman M., Kuck A.L., Norton H.W., 1986. Lactation curve of purebred and crossbred dairy cattle. J. Dairy Sci., 69, 195-203.

Grovum W.L., Williams V.J., 1973. Rate of passage of digesta in sheep. I. The effect of level of food intake on marker retention times along the small intestine and on apparent water absorption in the small and large intestines. Br. J. Nutr., 29, 13-21.

Hoden A., 1978. Rationnement au début de la lactation. In : La Vache laitière, 71-85, Ed. INRA Publications. Route de Saint-Cyr, 78000 Versailles, France.

Hoekstra J.A., 1986. A note on a partial adjustment model to describe lactation curves. Anim. Prod., 42, 431-432.

Huth Dr. F.W., 1971. Zur Frage des Verlaufes der Laktationskurve beim Rind. In : $\mathrm{X}^{\mathrm{e}}$ Congrès international de Zootechnie. Versailles, 1971, 7 pp.

Jenkins T.G., Ferrell C.L., 1982. Lactation curves of mature crossbred cows : comparison of four estimating functions. J. anim. Sci., 54, Suppl., 189 (Abstr.).

Jenkins T.G., Ferrell C.L., 1984. A note on lactation curves of crossbred cows. Anim. Prod., 39, 479-482. 
KALMAN R.E., 1960. A new approach to linear filtering and prediction problems. Trans. A.S.M.E. J. Basic Eng. Ser. D, 82, 34-45. Cité par Goodall \& Sprevak, 1985.

Kalman R.E., 1963. New methods of Wiener filtering theory. Proc. 1st Symp. Eng. Appns of Random Functions Theory and Prob. Ed. J.L. Bogdanoff and F. Kozin, 270-388. Wiley, New York. Cité par Goodall \& Sprevak, 1985.

Kalman R.E., BuCY R.S., 1961. New results in linear filtering and prediction problems. Trans A.S.M.E. J. Basic Eng. Ser. D, 83, 95-108. Cités par Goodall \& Sprevak, 1985.

Kellogg D.W., Urquhart N.S., Ortegia A.J., 1977. Estimating Holstein lactation curves with a Gamma curve. J. Dairy Sci., 60, 1308-1315.

Killen L., Kenne M., 1978. The shape of lactation curves in Irish dairy herds. Ir. J. Agric. Res., 17, $267-282$.

KrEFT R., 1967. Die Aufstellung eines mathematischen Modells der Milcheistung von Kühen wärhend der Laktation. Zesz, Nauk. W.S.R. walsznie, 23, 685-694. Cité par Panicke \& Bode, 1973.

Kuck A.L., Grossman M., Norton H.W., 1976. Studies of the lactation curve of purebred and crossbred dairy cattle. Program. 71st Ann. Meeting Am. Dairy Sci. Assoc., 131 (Abstr.). Cités par Grossman, Kuck \& Norton, 1986.

Kwizda R., Hopler E., Teufelhart N., 1957. Laktationsfaktoren zur Bestimmung der Milchleistung. Bodenkultur, Wien, 9, 335-350. Cités par Panicke \& Bode, 1973.

Madalena F.E., Martinez M.L., Freitas A.F., 1979. Lactation curves of Holstein-Friesian and Holstein-Friesian x Gir cows. Anim. Prod., 29, 101-107.

MAdSEN O., 1975. A comparison of some suggested measures of persistency of milk yield in dairy cows. Anim. Prod., 20, 191-197.

Malhotra P.K., Singh R.P., Singh R.N., 1980. Estimating lactation curve in Karan-Swiss cattle. Indian J. Anim. Sci., 50, 799-804.

MARQUARDT D.W., 1963. An algorithm for least squares estimation of non linear parameters. $J$. Soc. Appl. Math., 2, 431-441.

Maymone B., Malossini F., 1959. The rising phase of the lactation curve in dairy cows. Tierz. Zürcht. Biol., 73, 276-294. Cités par Decaen, Journet \& Poutous, 1970.

McMillan I.. Fitz-Earle M., Robson D.S., 1970. Quantitative genetics of fertility. I. Lifetime egg production of Drosophila melanogaster. Theoretical. Genetics, 65, 349-353. Cités par Gavora, Parker \& McMillan, 1971.

McMillan I, Fitz-Earle M., Butler L., Robson D.S., 1970. Quantitative genetics of fertility. II. Lifetime egg production of Drosophila melanogaster. Experimental. Genetics, 65, 355-369. Cités par Gavora, Parker \& McMillan, 1971.

McNally D.H., 1971. Mathematical model for poultry egg production. Biometrics, 27, 735-738. Cité par Gavora et al., 1982.

MıLoJic M., Simovic B., 1971. Lactation persistence of daughters from different bulls. In : $\mathrm{X}^{\mathrm{e}}$ Congrès international de Zootechnie, Versailles, 1971, 3 pp.

Minder C.E., MCMillan I., 1977. Estimation of compartmental model parameters using marginal likelihood. Biometrics, 33, 333-341.

Neal H.D. St. C., Thornley J.H.M., 1983. The lactation curve in cattle : a mathematical model of the mammary gland. J. Agric. Sci., Camb., 101, 389-400.

Nelder J.A., 1966. Inverse polynomials, a useful group of multi-factor response functions. Biometrics, 22, 128-141.

OstergaARd V., 1979. Strategies for concentrate feeding to attain optimum feeding level in high yielding dairy cows. Dairy Sci. Abstr., 41, $\mathrm{n}^{\circ} 5710$ (Abstr.).

Panicke L., Bode B., 1973. Mathematisches Modell des Laktationsverlaufes bei Milchkühen. Arch. Tierzucht., 16, 263-269.

Ponteconvo G., 1940. A study of the persistency in a herd of Ayrshire cows. J. Dairy Res., 11, 113.

Rao M.K., Sundaresan D., 1979. Influence of environment and heredity on the shape of lactation curves in Sahiwal cows. J. Agric. Sci., Camb., 92, 393-401.

Rowlands G.J., Lucey S., Russel A.M., 1982. A comparison of different models of the lactation curve in dairy cattle. Anim. Prod., 35, 135-144. 
Sauvant D., Fehr P.M., 1976. Modélisation et classification des types de courbes de composition et de production du lait de la chèvre. $27^{e}$ réunion annuelle de la F.E.Z., Zürich (Suisse), 2326 août 1976, Commission de production ovine et caprine.

Schafffer L.R., Minder C.E., McMillan I., Burnside E.B., 1977. Nonlinear techniques for predicting 305-day lactation production of Holsteins and Jerseys. J. Dairy Sci., 60, 1636-1644.

SchneEberger M., 1981. Inheritance of lactation curve in Swiss Brown cattle. J. Dairy Sci., 64, $475-483$

Shimizu H., UmRod S., 1976. An application of the weighted regression procedure for constructing the lactation curve in dairy cattle. Jap. J. Zootech. Sci., 47, 733-738.

Shimizu H., Umrod S., 1977. A note on the length of lactation and the number of consecutive days in average to obtain more typical lactation curve in dairy cattle. Jap. J. Zootech. Sci., 48, 273-280.

SikKa L.C., 1950. A study of the lactation as affected by heredity and environment. J. Dairy Res., 17, 231. Cité par Delage, Leroy \& Poly, 1953.

Singh B., Bhat P.N., 1978. Models of lactation curve in Hariana cattle. Indian J. Anim. Sci., 48, 791-797.

Singh B., Bhat P.N., Kumar R., 1979. Factors affecting the shape of the lactation curve in Hariana cattle. Indian J. Anim. Sci., 49, 495-498.

TURNER C.W., 1926. A quantitative form of expressing persistency of milk or fat secretion. $J$. Dairy Sci., 9, 203. Cité par Delage, Leroy \& Poly, 1953.

Turner C.W., Ragsdale A.C., Brody S., 1923. How the advance of the period of lactation affects the milk flow? J. Dairy Sci., 6, 527. Cités par Delage, Leroy \& Poly, 1953.

Vujicic I., Bacic B., 1961. (New equation of the lactation curve). Novi Sad. Ann. Sci. Agric., n 5. Cités par Wood, 1969.

Wood P.D.P., 1967.Algebraic model of the lactation curve in cattle. Nature, Lond., 216, 164-165.

Wood P.D.P., 1968. Factors affecting persistency of lactation in cattle. Nature, Lond., 218, 894.

Wood P.D.P., 1969. Factors affecting the shape of the lactation curve in cattle. Anim. Prod., 11, 307-316.

Woon P.D.P., 1970. A note on the repeatability of parameters of the lactation curve in cattle. Anim. Prod., 12, 535-538.

Wood P.D.P., 1972. A note on seasonal fluctuations in milk production. Anim. Prod., 15, 89-92.

Woon P.D.P., 1976. Algebraic models of the lactation curves of milk, fat and protein production, with estimates of seasonal variation. Anim. Prod., 22, 35-40.

Woon P.D.P., 1977. The biometry of lactation. J. Agric. Sci., Camb. 88, 333-339.

WooD P.D.P., 1979. A simple model of lactation curves for milk yield, food requirement and body weight. Anim. Prod., 28, 55-63.

Woon P.D.P., 1980. Breed variations in the shape of the lactation curve of cattle and their implications for efficiency. Anim. Prod., 31, 133-141.

Zimmermann E., Sommer H., 1973. Zum Laktationsverlauf von Kühen in Hochleistungsherden und dessen Beeinflussung durch nichterbliche Faktoren. Züchtungskunde, 45, 75-88. 